\title{
Neutrino mass from Higgs quadruplet and multicharged Higgs searches at the LHC
}

\author{
Tathagata Ghosh, ${ }^{1,2, *}$ Sudip Jana, ${ }^{2,3, \dagger}$ and S. Nandi ${ }^{2, *}$ \\ ${ }^{1}$ Department of Physics and Astronomy, University of Hawaii, Honolulu, Hawaii 96822, USA \\ ${ }^{2}$ Department of Physics and Oklahoma Center for High Energy Physics, Oklahoma State University, \\ Stillwater, Oklahoma 74078-3072, USA \\ ${ }^{3}$ Theoretical Physics Department, Fermilab, Batavia, Illinois 60510, USA
}

(Received 5 March 2018; published 20 June 2018)

\begin{abstract}
In this paper, we revisit the dimension-7 neutrino mass generation mechanism based on the addition of an isospin $3 / 2$ scalar quadruplet and two vectorlike isotriplet leptons to the standard model. We discuss the LHC phenomenology of the charged scalars of this model, complemented by the electroweak precision and lepton flavor violation constraints. We pay particular attention to the triply charged and doubly charged components. We focus on the same-sign-trilepton signatures originating from the triply charged scalars and find a discovery reach of $600-950 \mathrm{GeV}$ at $3 \mathrm{ab}^{-1}$ of integrated luminosity at the LHC. On the other hand, doubly charged Higgs has been an object of collider searches for a long time, and we show how the present bounds on its mass depend on the particle spectrum of the theory. Strong constraints on the model parameter space can arise from the measured decay rate of the standard model Higgs to a pair of photons as well.
\end{abstract}

DOI: 10.1103/PhysRevD.97.115037

\section{INTRODUCTION}

The remarkable discovery of the $125 \mathrm{GeV}$ scalar particle by the CMS and ATLAS collaborations [1,2] is the crowning achievement of the Run-I of the LHC. The data collected by the LHC experiments so far indicate that the discovered particle is the final piece of the standard model (SM) - the Higgs boson, which provides mass to the fermions and gauge bosons of the SM via spontaneous symmetry breaking. At the same time, any signature beyond the SM remains elusive at the LHC. Notwithstanding many successes of the SM, it fails to answer many critical questions. Hence, the pursuit of unearthing signals of new physics has been at the forefront of particle physics experiments for many decades.

Among the most robust evidence that points out to an important inadequacy of the SM is the existence of nonzero tiny masses of neutrinos. The neutrinos are the only class of fermion within the SM, the mass of which cannot be generated by the Higgs mechanism, due to the absence of right-handed neutrinos. However, various neutrino oscillation experiments have long established the fact that not

\footnotetext{
tghosh@hawaii.edu

†sudip.jana@okstate.edu

s.nandi@okstate.edu
}

Published by the American Physical Society under the terms of the Creative Commons Attribution 4.0 International license. Further distribution of this work must maintain attribution to the author(s) and the published article's title, journal citation, and DOI. Funded by SCOAP. only do neutrinos possess small masses $[\mathcal{O}(0.01-0.1 \mathrm{eV})]$ but also they mix between flavors. In addition, the Planck Collaboration constrains the sum of neutrino masses to be $\sum m_{i} \lesssim 0.23 \mathrm{eV}$ [3], which again emphasizes the fact that neutrino masses are many orders of magnitude smaller than their charged lepton counterparts. This drastic departure of neutrino masses and mixings from charged leptons poses a fundamental question: how can such tiny neutrino masses be generated?

The simplest way to achieve that goal is via an effective dimension-5 operator, $L L H H / M$ [4], where $H$ is the SM Higgs doublet, $L$ is the left-handed lepton doublet, and $M$ is the scale of new physics. Under this mechanism, neutrinos acquire a mass $m_{\nu} \sim v^{2} / M$, with $v$ being the vacuum expectation value (VEV) of $H$. There have been many realizations of such dimension-5 operators in the literature, namely, Type-I seesaw [5], Type-II seesaw [6], Type-III seesaw [7], loop induced [8], etc., with all new particles being at the mass order $M$. From the above formula of neutrino masses, one can notice that neutrino oscillation data, combined with cosmological constraint, will force $M \sim \mathcal{O}\left(10^{14}-10^{15}\right) \mathrm{GeV}$ with $\mathcal{O}(1)$ Yukawa couplings. Alternatively, one needs an unusually small Yukawa coupling, $Y_{\nu} \sim 10^{-6}$, for $\mathrm{TeV}$-scale $M$. In either case, the LHC is unlikely to probe any signature of such particles. Instead, we focus on a model proposed by Babu et al. (BNT) [9], where neutrino masses are generated at tree level by an effective dimension-7 operator, $L L H H\left(H^{\dagger} H\right) / M^{3}$, resulting in a neutrino mass formula, $m_{\nu} \sim v^{4} / M^{3}$. Owing to the 
increased suppression factor $M^{3}$ in the denominator, one can easily lower the scale of new physics in this model to $\mathrm{TeV}$ without introducing minuscule Yukawa couplings. The above model contains two vectorlike lepton triplets $\left(\Sigma_{1,2}\right)$ and an isospin $\frac{3}{2}$ scalar quadruplet $(\Delta)$ on top of the SM fields. Hence, this model predicts striking same-sign multilepton signatures at the LHC due to the presence of multicharged scalars and vectorlike leptons. Scalar quadruplets can be very interesting for electroweak phase transition as well [10].

The goal of our paper is twofold. First, we present a detailed analysis of electroweak precision test (EWPT) constraints on the Higgs spectrum of the model for the first time. Next, we investigate the latest LHC and lepton flavor violation (LFV) bounds on the Higgs sector, not ruled out by the EWPT, and further project the future LHC reach of the triply charged Higgs boson for definitive validation/ falsification of the model.

References [11-14] have studied the BNT model in the context of the LHC and dark matter previously. Nonetheless, the LHC experiments have accumulated a significant volume of data since then, and a revision of those constraints from the new data is warranted at this point. In addition, a loop-induced dimension- 5 operator is also present in the model, which contributes to the neutrino mass generation at a comparable rate with respect to the dimension-7 operator for $M_{\Sigma} \gtrsim \mathcal{O}(\mathrm{TeV})$. Although the existence of this dimension-5 operator is well known $[9,11]$, the impact of dimension-5 operator interplay with the dimension-7 operator on the LHC searches was not taken into account in previous studies at a quantitative level.

In addition, we would like to point out that the LHC experiments traditionally show their bound on doubly charged Higgs particle mass in same-sign dilepton final states assuming a $100 \%$ branching ratio (BR) for particular flavor combinations. Instead, we reinterpret their results using realistic benchmark points (BP), consistent with neutrino oscillation data, and show that the constraints on doubly charged Higgs mass can be relaxed. Also, we demonstrate that for our realistic BPs the proper decay length of doubly and triply charged Higgs bosons are quite large in regions of the parameter space, and we discuss when they will be beyond the scope of prompt lepton searches performed at the LHC.

LFV constraints on the model were previously discussed in Ref. [15] for very light $\Sigma_{1,2}(\sim 200 \mathrm{GeV})$, and the authors did not take into account the contribution of multicharged scalars on LFV processes. In contrast, we derive relevant LFV constraints due to light scalars $\left(M_{\Delta} \lesssim 1 \mathrm{TeV}\right)$. In our chosen benchmark scenarios, $\Sigma_{1,2}$ are much heavier $(\sim 5 \mathrm{TeV})$ than $H, \Delta$, which in turn forces their contribution to LFV processes to be negligible. Using the current most stringent bound by the MEG Collaboration [16], a lower bound on induced VEV $v_{\Delta}$ as a function of mass $M_{\Delta}$ has been derived. A similar study has been performed for a
Higgs triplet by Ref. [17] within the Type-II seesaw framework.

Lastly, we search for the triply charged Higgs boson at the LHC in the same-sign three leptons final state. A potential discovery of $\Delta^{ \pm \pm \pm}$at the LHC will shed some light on the possible mechanism of neutrino mass generation.

The paper is organized as follows. In Sec. II, we present a brief overview of the BNT model and the neutrino mass generation mechanisms within the model, along with our choice of neutrino oscillation parameters for subsequent calculations. In Secs. III and IV, we discuss EWPT and LFV constraints, respectively, on the Higgs sector of the model. Updated constraints form various LHC searches relevant to the Higgs sector of this model are discussed in Sec. V. We also outline the projected reach at the LHC for triply charged Higgs in the same section, in association with detailed discussion on their relevant production and decay mechanisms. Finally, we conclude in Sec. VI.

\section{MODEL AND FORMALISM}

In this section, we present a brief overview of the BNT model [9]. The chief goal of the model is to develop light neutrino masses with new physics at the $\mathrm{TeV}$ scale without introducing unnaturally small Yukawa couplings or finetuned cancellations. The BNT model is based on the SM symmetry group $S U(3)_{C} \times S U(2)_{L} \times U(1)_{Y}$. The enlarged particle content of the model includes an isospin $\frac{3}{2}$ scalar quadruplet, $\Delta$, and a pair of vectorlike fermion triplets, $\Sigma_{1,2}$. We use $H$ to denote the SM-like Higgs doublet. The particle contents along with their quantum numbers are shown in Table I below.

\section{A. Higgs sector of the model}

The scalar kinetic and potential terms of the model are given by

TABLE I. Matter, gauge, and Higgs contents of the BNT model.

\begin{tabular}{lc}
\hline \hline Matter & $S U(3)_{C} \times S U(2)_{L} \times U(1)_{Y}$ \\
& $\left(\begin{array}{l}u \\
d\end{array}\right)_{L} \sim\left(3,2, \frac{1}{3}\right), u_{R} \sim\left(3,1, \frac{4}{3}\right), d_{R} \sim\left(3,1,-\frac{2}{3}\right)$ \\
& $\left(\begin{array}{c}\nu_{e} \\
e\end{array}\right)_{L} \sim(1,2,-1), e_{R} \sim(1,1,-2)$ \\
& $\Sigma_{2} \equiv\left(\begin{array}{c}\Sigma_{2}^{++} \\
\Sigma_{2}^{+} \\
\Sigma_{2}^{0} \\
\square\end{array}\right) \sim(1,3,2), \Sigma_{1} \equiv\left(\begin{array}{c}\Sigma_{1}^{++} \\
\Sigma_{1}^{+} \\
\Sigma_{1}^{0}\end{array}\right) \sim(1,3,2)$ \\
Gauge & $G_{a, a=1-8}^{\mu}, A_{i, i=1-3}^{\mu}, B^{\mu}$ \\
Higgs $\quad H \equiv\left(\begin{array}{c}\Delta^{+++} \\
\Delta^{++} \\
\Delta^{+} \\
\Delta^{0} \\
\square\end{array}\right) \sim(1,4,3)$ \\
\hline \hline
\end{tabular}




$$
\mathcal{L}_{\text {Scalar }}^{\text {Kin }}=\left(D^{\mu} \Delta\right)^{\dagger}\left(D_{\mu} \Delta\right)+\left(D^{\mu} H\right)^{\dagger}\left(D_{\mu} H\right)+V(H, \Delta),
$$

with the covariant derivatives

$$
\begin{aligned}
& D_{\mu} H=\left(\partial_{\mu}-i g \vec{\tau} \cdot \vec{W}_{\mu}-i g^{\prime} \frac{Y}{2} B_{\mu}\right) H, \\
& D_{\mu} \Delta=\left(\partial_{\mu}-i g \vec{T} \cdot \vec{W}_{\mu}-i g^{\prime} \frac{Y}{2} B_{\mu}\right) \Delta,
\end{aligned}
$$

where $\vec{\tau}$ are standard Pauli matrices and $\vec{T}$ are $S U(2)$ generators in the isospin $\frac{3}{2}$ representation [11]. The interactions of the new scalar field $\Delta$ with the gauge bosons originate from the above term. The most general renormalizable scalar potential involving the Higgs fields of the model is given by

$$
\begin{aligned}
V(H, \Delta)= & -\mu_{H}^{2} H^{\dagger} H+\mu_{\Delta}^{2} \Delta^{\dagger} \Delta+\lambda_{1}\left(H^{\dagger} H\right)^{2}+\lambda_{2}\left(\Delta^{\dagger} \Delta\right)^{2} \\
& +\lambda_{3}\left(H^{\dagger} H\right)\left(\Delta^{\dagger} \Delta\right)+\lambda_{4}\left(H^{\dagger} \tau_{a} H\right)\left(\Delta^{\dagger} T_{a} \Delta\right) \\
& +\left\{\lambda_{5} H^{3} \Delta^{\star}+\text { H.c. }\right\} .
\end{aligned}
$$

We assume $\mu_{\Delta}^{2}>0$, and thus $\Delta$ cannot initiate any spontaneous symmetry breaking. Hence, similar to the $\mathrm{SM}$, the electroweak (EW) symmetry is broken spontaneously once the Higgs doublet, $H$, acquires a VEV, $\langle H\rangle=\frac{v_{H}}{\sqrt{2}}$. Interestingly, even with a positive $\mu_{\Delta}{ }^{2}$, due to the presence of the $\lambda_{5}$ term in the potential, the neutral component of $\Delta$ acquires an induced VEV at the tree level,

$$
\langle\Delta\rangle=\frac{v_{\Delta}}{\sqrt{2}}=-\frac{\lambda_{5} v_{H}^{3}}{2 \sqrt{2} M_{\Delta}^{2}} .
$$

However, $v_{\Delta}$ suffers from strong bounds coming from the EW $\rho$ parameter. In the BNT model, the analytical form of the $\rho$ parameter at tree level is $\rho \approx\left(1-6 v_{\Delta}^{2} / v_{H}^{2}\right)$. In order to satisfy the experimentally observed value, $\rho=$ $1.00037_{-0.00023}^{+0.00023}$ [18] at $2 \sigma, v_{\Delta}$ is constrained to be $v_{\Delta} \lesssim 1 \mathrm{GeV}$. In the above equation $M_{\Delta}$ denotes the mass of the neutral scalar $\Delta^{0}$, which can be expressed as

$$
M_{\Delta}^{2}=\mu_{\Delta}^{2}+\frac{v_{H}^{2}}{8}\left(4 \lambda_{3}+3 \lambda_{4}\right)
$$

On the other hand, masses of other members of the $\Delta$ quadruplet are given by

$$
M_{i}^{2}=M_{\Delta}^{2}-Q_{i} \frac{\lambda_{4}}{4} v_{H}^{2},
$$

where $Q_{i}$ is the (non-negative) electric charge of the respective field. We neglect small corrections proportional to $v_{\Delta}$ in the above mass-squared expressions since $v_{\Delta} \ll v_{H}$. The mass gaps are equally spaced. Also, two mass orderings are possible here. For $\lambda_{4}$ positive, we have the ordering $M_{\Delta^{+++}}<M_{\Delta^{++}}<M_{\Delta^{+}}<M_{\Delta^{0}}$, and for $\lambda_{4}$ negative, we have the ordering $M_{\Delta^{+++}}>M_{\Delta^{++}}>M_{\Delta^{+}}>M_{\Delta^{0}}$. Clearly, large mass gaps between the constituents of the quadruplet can be developed by choosing a large value of $\lambda_{4}$ that is allowed by perturbativity. These mass splittings are an integral part of our present analysis. We shall see in subsequent sections that not only do they play a pivotal role in EW precision constraints but also LHC mass reaches are highly dependent on them.

\section{B. Boundedness of the Higgs potential}

When the scalar fields become large in any direction of the field space, a necessary condition for the vacuum stability comes from requiring that the potential given in Eq. (2.3) be bounded from below [19-21]. Obviously, at large field values, the potential (2.3) is generically dominated by the part containing the quartic terms, and hence the study of $V^{(4)}(H, \Delta)$ [cf. Eq. (2.7)] will be sufficient to obtain the main boundedness constraints,

$$
\begin{aligned}
V^{(4)}(H, \Delta)= & \lambda_{1}\left(H^{\dagger} H\right)^{2}+\lambda_{2}\left(\Delta^{\dagger} \Delta\right)^{2}+\lambda_{3}\left(H^{\dagger} H\right)\left(\Delta^{\dagger} \Delta\right) \\
& +\lambda_{4}\left(H^{\dagger} \tau_{a} H\right)\left(\Delta^{\dagger} T_{a} \Delta\right)+\left\{\lambda_{5} H^{3} \Delta^{\star}+\text { H.c. }\right\} .
\end{aligned}
$$

Now, we can pick up specific field directions to obtain the conditions. In the absence of any coupling between doublet and triplet Higgs bosons, i.e., $\lambda_{3}=\lambda_{4}=\lambda_{5}=0$, it is obvious that $\lambda_{1}>0 \& \lambda_{2}>0$ will ensure that the potential is bounded from below. On the other hand, if we set $\lambda_{5}$ to zero ${ }^{1}$ for simplicity, then the vacuum stability conditions become

$\lambda_{1}>0, \quad \lambda_{2}>0, \quad 4 \lambda_{3}+3 \lambda_{4}+4 \sqrt{\lambda_{1} \lambda_{2}}>0$,

which can be simplified to

$\lambda_{1}>0, \quad \lambda_{2}>0, \quad \lambda_{4}>-\frac{4}{3}\left(\lambda_{3}+\sqrt{\lambda_{1} \lambda_{2}}\right)$.

For a realistic choice of a benchmark point $\lambda_{1,2,3}=$ $(0.13,0.25,1.37)$ and $v_{\Delta}=10^{-4} \mathrm{GeV}$, which gives us the masses of $C P$ even Higgs bosons to be 125 and $400 \mathrm{GeV}$, respectively (mixing among them is assumed to be tiny), we find from the above equation that $\lambda_{4}>-2.07$. In other words, for the above benchmark point, we obtain a bound

\footnotetext{
${ }^{1}$ It is important to mention that we have also done the full analysis to get the boundedness constraints taking nonzero values of $\lambda_{5}$. However, the relations obtained from that analysis are complex and not insightful. Hence, to get a compact form of boundedness criteria, we set $\lambda_{5}$ to zero. It will not affect the phenomenology we are concentrating on in this paper since $\lambda_{5}$ is always taken to be small [cf. Eq. (2.4)] to get correct order neutrino mass, with $M_{\Delta} \sim \mathcal{O}(100-1000) \mathrm{GeV}$.
} 
on the mass splitting among the quadruplet members to be $\Delta M \gtrsim-35 \mathrm{GeV}$. We will show in Sec. III that a stronger limit on $\Delta M$ can be derived from EWPT for this benchmark point.

\section{Generation of neutrino mass}

Neutrino masses arise in the model from the renormalizable Lagrangian [9]

$\mathcal{L}_{\nu \text {-mass }}=Y_{i} \overline{L_{i L}}{ }^{c} H^{*} \Sigma_{1}+Y_{i}^{\prime} \overline{\Sigma_{2}} \Delta L_{i L}+M_{\Sigma} \overline{\Sigma_{2}} \Sigma_{1}+$ H.c.,

where $Y_{i}, Y^{\prime}{ }_{i}$ are Yukawa couplings and $i$ is the generation index. Integrating out the $\Sigma_{1,2}$ fermions, one obtains an effective dimension-5 neutrino mass operator

$$
\mathcal{L}_{\text {eff }}=-\frac{\left(Y_{i} Y_{j}^{\prime}+Y_{j} Y_{i}^{\prime}\right) \overline{L_{i L}}{ }^{2} L_{j L} H^{*} \Delta}{M_{\Sigma}}+\text { H.c.. }
$$

The tree-level diagram generating this operator is shown in Fig. 1 [9]. The detailed structure of the Yukawa interactions is given in Ref. [11].

We have already seen from the analysis of the Higgs potential that $\Delta^{0}$ acquires an induced VEV $v_{\Delta}=-\lambda_{5} v^{3} / 2 M_{\Delta}^{2}$. When this value is substituted in Eq. (2.11), to the leading order, we obtain the neutrino masses at tree level, $\left(m_{\nu}\right)^{\text {tree }}$, which can be written as [9]

$\left(m_{\nu}\right)_{i j}^{\mathrm{tree}}=-\frac{\left(Y_{i} Y_{j}^{\prime}+Y_{i}^{\prime} Y_{j}\right) v_{\Delta} v_{H}}{M_{\Sigma}}=\frac{\lambda_{5}\left(Y_{i} Y_{j}^{\prime}+Y_{i}^{\prime} Y_{j}\right) v_{H}^{4}}{2 M_{\Sigma} M_{\Delta^{0}}^{2}}$.

This provides us with a tree-level dimension-7 neutrino mass generation mechanism. Clearly, the particle content of the model prevents it from developing a dimension-5 operator at the tree level. Nevertheless, there is no mechanism present in the model that prevents generating a dimension-5 operator at the loop level. For the diagram that generates the loop-level dimension-5 operator, we refer the reader to Fig. 2 [11,12]. The loop contribution to the

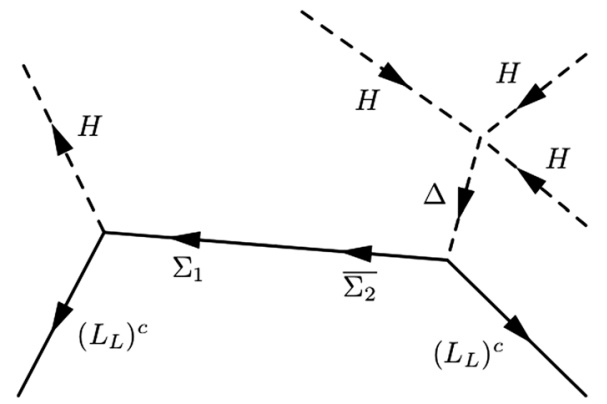

FIG. 1. Tree-level diagram that generates dimension-7 operator for neutrino mass.

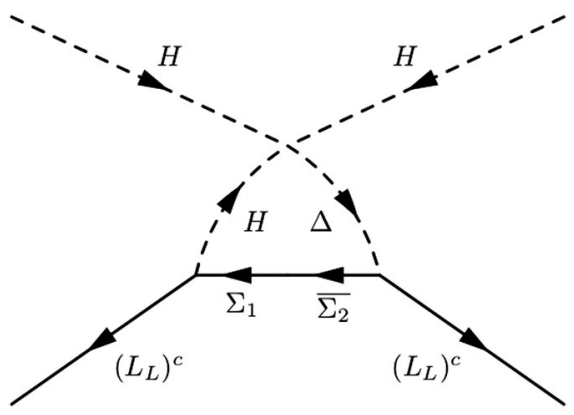

FIG. 2. Loop-level diagram that generates the dimension-5 operator for neutrino mass.

neutrino mass, $\left(m_{\nu}\right)^{\text {loop }}$, can be computed at the leading order $\left[\mathcal{O}\left(v_{H}^{2}\right)\right]$ as [11]

$$
\begin{aligned}
\left(m_{\nu}\right)_{i j}^{\text {loop }}= & \frac{(3+\sqrt{3}) \lambda_{5} v_{H}^{2} M_{\Sigma}\left(Y_{i} Y_{j}^{\prime}+Y_{i}^{\prime} Y_{j}\right)}{32 \pi^{2}\left(M_{\Delta}^{2}-M_{H}^{2}\right)} \\
& \times\left(\frac{M_{\Delta}^{2} \log \left(\frac{M_{\Sigma}^{2}}{M_{\Delta}^{2}}\right)}{M_{\Sigma}^{2}-M_{\Delta}^{2}}-\frac{M_{H}^{2} \log \left(\frac{M_{\Sigma}^{2}}{M_{H}^{2}}\right)}{M_{\Sigma}^{2}-M_{H}^{2}}\right) .
\end{aligned}
$$

It is important to examine what the relevant masses $M_{\Delta}$ and $M_{\Sigma}$ are that determine the relative contribution of the loop-level dimension-5 operator in comparison with the tree-level dimension-7 operator. In Fig. 3, we plot $\left(m_{\nu}\right)_{i j}^{\text {loop }} /\left(m_{\nu}\right)_{i j}^{\text {tree }}$ as a function of $M_{\Delta}$ for three different values of $M_{\Sigma}$. We should mention here that both $\Delta^{0}$ and $\Delta^{ \pm}$ enter the loop-level dimension-5 operator of Eq. (2.13) [11], but they are assumed to be the same in the computation of Fig. 3 for simplicity. For $M_{\Sigma}=0.5,1 \mathrm{TeV},\left(m_{\nu}\right)_{i j}^{\text {tree }}$

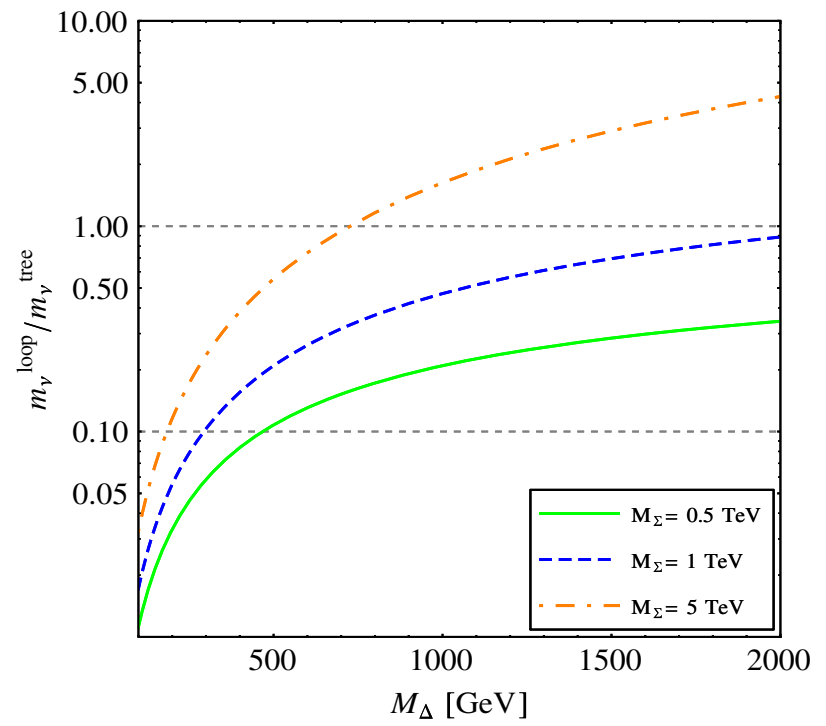

FIG. 3. $\left(m_{\nu}\right)_{i j}^{\text {loop }} /\left(m_{\nu}\right)_{i j}^{\text {tree }}$ as a function of $M_{\Delta}$ for different values of $M_{\Sigma}$. 
dominates over $\left(m_{\nu}\right)_{i j}^{\text {loop }}$ in the range of $M_{\Delta} \lesssim 2 \mathrm{TeV}$. In contrast, for $M_{\Sigma}=5 \mathrm{TeV},\left(m_{\nu}\right)_{i j}^{\text {loop }}$ catches up with $\left(m_{\nu}\right)_{i j}^{\text {tree }}$ at $M_{\Delta} \sim 0.75 \mathrm{TeV}$. Thus, it is desirable to set $M_{\Sigma} \lesssim 1 \mathrm{TeV}$ to test purely dimension-7 generation of neutrino mass at the LHC. However, such a choice of the parameter will significantly increase the difficulty of signal simulation for LHC searches. This is due to the fact that in the aforementioned scenario we shall not be able to integrate out $M_{\Sigma}$ and a very careful and tedious treatment is needed regarding the charged lepton mass matrix without any significant phenomenological gain at the LHC. On the other hand, for $M_{\Sigma} \sim 5 \mathrm{TeV}$, we can avoid this complexity and perform relevant collider simulations with ease. In addition, in the range of $M_{\Delta}$ that is accessible for the ongoing run of the LHC, as will be shown in Sec. VE, the dimension-7 operator is still dominant with $M_{\Sigma} \sim 5 \mathrm{TeV}$. Also, we should emphasize here that our main goal in this paper is to study multiple aspects of the Higgs sector of the BNT model. Various Higgs analyses performed in this paper are, to a large extent, not sensitive to dimension-7 or dimension-5 neutrino mass generation operators. They can only alter the leptonic decay BRs of Higgs bosons marginally and will not qualitatively impact the important conclusions of this study. Henceforth, we set $M_{\Sigma}=5 \mathrm{TeV}$ for the rest of the paper.

We conclude this subsection with a brief remark on possible extensions of the BNT model, available in the literature, that can potentially prevent the appearance of a dimension-5 operator via loops. One way to achieve that is to impose a symmetry that forbids the generation of neutrino masses at dimensions $d<7$. In effective field theory language, the dimension- 5 and dimension- 7 operators can be written as $\mathcal{O}^{5}=L L H H$ and $\mathcal{O}^{7}=$ $L L H H\left(H^{\dagger} H\right)$, respectively. Similarly, one can expand it further by adding higher powers of the combination $\left(H^{\dagger} H\right)$ to generate $d>7$ dimension operators. The shortcoming of this approach is that $\left(H^{\dagger} H\right)$ is a singlet under any symmetry and does not carry any charge. Thus, one cannot avert the problem, and all powers of $\left(H^{\dagger} H\right)$ are allowed. Therefore, we need to add (a) new Higgs field(s) to the theory and charge it (them)

$$
U_{P M N S}=\left(\begin{array}{c}
c_{12} c_{13} \\
-c_{23} s_{12}-s_{23} s_{13} c_{12} e^{i \delta} \\
s_{23} s_{12}-c_{23} s_{13} c_{12} e^{i \delta}
\end{array}\right.
$$

with $c_{i j}\left(s_{i j}\right)=\cos \theta_{i j}\left(\sin \theta_{i j}\right)$, and $P=\operatorname{diag}\left(1, e^{i \alpha_{1}}, e^{i \alpha_{2}}\right)$.

In the BNT model, due to the presence of two vectorlike lepton triplets, the neutral lepton mass matrix is $5 \times 5$ with rank 4 [11] at tree level. Therefore, at that order, the under some $U(1)$ or discrete symmetry that allows a dimension-7 operator but not any operator of lower dimensions.

In the context of the BNT model, one can add another Higgs doublet to the field, similar to the two Higgs doublet model $[22,23]$, leading to the following effective Lagrangian in the $n$th dimension:

$\mathcal{L}_{\text {eff }}^{d=2 n+5}=\frac{1}{\Lambda_{\mathrm{NP}}^{d-4}}\left(L L H_{u} H_{u}\right)\left(H_{u} H_{d}\right)^{n}, \quad n=1,2,3, \ldots$

The simplest pure dimension-7 model can be constructed from this effective Lagrangian by introducing a $Z_{5}$ symmetry and assigning the following charges [24]:

$$
\begin{aligned}
q_{H_{u}} & =0, & q_{H_{d}}=3, & q_{L}=1,
\end{aligned}
$$

One can also attain the same goal by using one Higgs doublet only and a singlet scalar [24]. A more complex solution is realized within the next-to-minimal supersymmetry (SUSY) standard model, which contains two Higgs doublets and a singlet [25]. Finally, if one is interested in pure dimension-7 loop-induced neutrino mass generation, he/she can take a look at Ref. [26].

\section{Neutrino mass hierarchies and Yukawa couplings}

Next, we discuss the benchmark Yukawa couplings we used in our paper, consistent with all neutrino mass and mixing data. In a basis where the charged lepton mass matrix is diagonal, the light neutrino matrix $\left(m_{\nu}\right)$ can be diagonalized as

$\left(m_{\nu}\right)^{\operatorname{diag}}=\operatorname{diag}\left(m_{1}, m_{2}, m_{3}\right)=U_{P M N S}^{T} m_{\nu} U_{P M N S}$,

where $U_{P M N S}$ is the neutrino mixing matrix. $U_{P M N S}$ is parametrized by three mixing angles $\theta_{i j}(i j=12,13,23)$, one Dirac phase $(\delta)$, and two Majorana phases $\left(\alpha_{1,2}\right)$ as

$\left.\begin{array}{cc}s_{12} c_{13} & s_{13} e^{-i \delta} \\ c_{23} c_{12}-s_{23} s_{13} s_{12} e^{i \delta} & s_{23} c_{13} \\ -s_{23} c_{12}-c_{23} s_{13} s_{12} e^{i \delta} & c_{23} c_{13}\end{array}\right) P$,

neutrino mass spectrum consists of one massless neutrino, two massive light neutrinos, and two heavy neutrinos, which are nearly degenerate. Since the lightest neutrino in the model is massless at tree level, we can approximately 
TABLE II. The best-fit values and $3 \sigma$ ranges of neutrino oscillation parameters, extracted from the global analysis of Ref. [28]. We show our choice of these parameters, used for the rest of the paper, in the last column. Please note that $\Delta m_{3 l}^{2} \equiv \Delta m_{32}^{2}>0$ for $\mathrm{NH}$ and $\Delta m_{3 l}^{2} \equiv \Delta m_{31}^{2}<0$ for $\mathrm{IH}$.

\begin{tabular}{lccc}
\hline \hline Oscillation parameter & Best fit & $3 \sigma$ range & Our benchmark \\
\hline$\Delta m_{21}^{2}\left(10^{-5} \mathrm{eV}^{2}\right)$ & 7.50 & $7.02 \rightarrow 8.09$ & 7.50 \\
$\Delta m_{3 l}^{2}\left(10^{-3} \mathrm{eV}^{2}\right)$ & $2.457(\mathrm{NH})$ & $2.317 \rightarrow 2.607(\mathrm{NH})$ & 2.50 \\
& $-2.449(\mathrm{IH})$ & $-2.590 \rightarrow-2.307(\mathrm{IH})$ & -2.50 \\
$\sin ^{2} \theta_{12}$ & 0.304 & $0.270 \rightarrow 0.344$ & 0.320 \\
$\sin ^{2} \theta_{23}$ & $0.452(\mathrm{NH})$ & $0.382 \rightarrow 0.643(\mathrm{NH})$ & 0.500 \\
$\sin ^{2} \theta_{13}$ & $0.579(\mathrm{IH})$ & $0.389 \rightarrow 0.644(\mathrm{IH})$ & 0.0250 \\
$\delta$ & $0.0218(\mathrm{NH})$ & $0.0186 \rightarrow 0.0250(\mathrm{NH})$ & \multirow{2}{*}{0} \\
& $0.0219(\mathrm{IH})$ & $0.0188 \rightarrow 0.0251(\mathrm{IH})$ & \\
\hline \hline
\end{tabular}

estimate the mass eigenvalues of two light massive neutrinos in terms of the solar and atmospheric mass-squared differences as ${ }^{2}$

(i) Normal hierarchy $(\mathrm{NH}), m_{1} \ll m_{2} \approx m_{3}$ :

$$
\begin{aligned}
& m_{1}=0, \quad m_{2}=\sqrt{\Delta m_{21}^{2}}, \\
& m_{3}=\sqrt{\Delta m_{32}^{2}+\Delta m_{21}^{2}},
\end{aligned}
$$

(ii) Inverted hierarchy (IH), $m_{3} \ll m_{1} \approx m_{2}$ :

$$
m_{3}=0, \quad m_{1}=\sqrt{\Delta m_{13}^{2}}, \quad m_{2}=\sqrt{\Delta m_{13}^{2}+\Delta m_{21}^{2}},
$$

where $\Delta m_{i j}^{2} \equiv m_{j}^{2}-m_{i}^{2}$. The best-fit values and $3 \sigma$ ranges of oscillation parameters, extracted from Ref. [28], are tabulated in Table II. We also show, in the same table, the benchmark values of these parameters that we shall use for the rest of the paper.

A comment is in order here regarding the $C P$-violating phases. These phases certainly have some impact on the Yukawa couplings, especially on the off-diagonal elements of the Yukawa matrix. However, the only off-diagonal

\footnotetext{
${ }^{2}$ A change in the lightest neutrino mass can change the relative strength of Yukawa couplings of the quadruplet members with leptons, which in turn will change the decay BRs of $\Delta^{ \pm \pm}$and $\Delta^{ \pm \pm \pm}$in different lepton flavor combinations. When $m_{\nu}^{\text {lightest }} \gtrsim 0.1 \mathrm{eV}, \Delta^{ \pm \pm}$decay BRs to flavor diagonal leptonic pairs change significantly from the benchmark scenarios considered here and tend to converge to similar values, while the offdiagonal channel BRs remain small. Although in the BNT model the lightest neutrino will always be massless at tree level, at oneloop level, it will acquire a small mass $\mathcal{O}\left(10^{-18}\right) \mathrm{eV}$ from the new physics contributions of the BNT model. In addition, at two-loop level, the SM itself provides an even larger correction of $\mathcal{O}\left(10^{-14}\right)$ eV [27], assuming $M_{N P} \sim \mathcal{O}(\mathrm{TeV})$. However, still, these quantum corrections are too small to have any observable change in the Yukawa couplings, and we find it safe to assume $m_{\nu}^{\text {lightest }}=0$ for our calculations.
}

channel that has a coupling that is comparable to or larger than $\mu \mu(e e)$ for $\mathrm{NH}(\mathrm{IH})$ is $e \tau$, and this particular channel is not interesting in the context of the LHC owing to the poor $\tau$ identification efficiency. $e \mu$ and $e \tau$ always remain small in both hierarchy scenarios. Consequently, these phases have no tangible impact on our phenomenological analyses. Hence, we set all $C P$-violating phases to be 0 , for simplicity, in our analysis.

\section{ELECTROWEAK PRECISION TESTS}

In this section, we put our effective theory, after integrating out $M_{\Sigma}$, under the microscope of high precision EW observables measured at the LEP and Stanford linear collider. For heavy $\Sigma_{1,2}$, the Higgs quadruplet, $\Delta$, only contributes to processes that can distort successful EW predictions of the SM. The principal effect of the $S U(2)_{L}$ quadruplet on the EW observables enters by means of oblique parameters, which are nothing but the gauge boson vacuum polarization correlations [29]. The oblique parameters are parametrized by three independent parameters $S, T$, and $U$ defined as [29]

$$
\begin{aligned}
\alpha S & \equiv 4 e^{2}\left[\Pi_{33}^{\prime}(0)-\Pi_{3 Q}^{\prime}(0)\right] \\
\alpha T & \equiv \frac{e^{2}}{s_{W}^{2} c_{W}^{2} M_{Z}^{2}}\left[\Pi_{11}(0)-\Pi_{33}(0)\right] \\
\alpha U & \equiv 4 e^{2}\left[\Pi_{11}^{\prime}(0)-\Pi_{33}^{\prime}(0)\right],
\end{aligned}
$$

where $\alpha$ is the fine structure constant and $s_{W}\left(c_{W}\right)$ are the sine (cosine) of the EW mixing angle. $\Pi_{X Y}(X, Y=1,3, Q)$ represents the vacuum polarization amplitudes, and $\Pi_{X Y}^{\prime}=\frac{d}{d q^{2}} \Pi_{X Y}\left(q^{2}\right)$.

Here, we make use of the general formulas of Ref. [30] in the quadruplet. Two important assumptions made in the calculation of Ref. [30] are (i) the complex scalar multiplet of interest does not acquire any VEV and (ii) its members do not mix with themselves or any other scalar. In the BNT model, it is natural to work in a limit where $v_{\Delta} \lesssim \mathcal{O}(1) \mathrm{GeV}$. Otherwise, we shall require either $Y$ or 


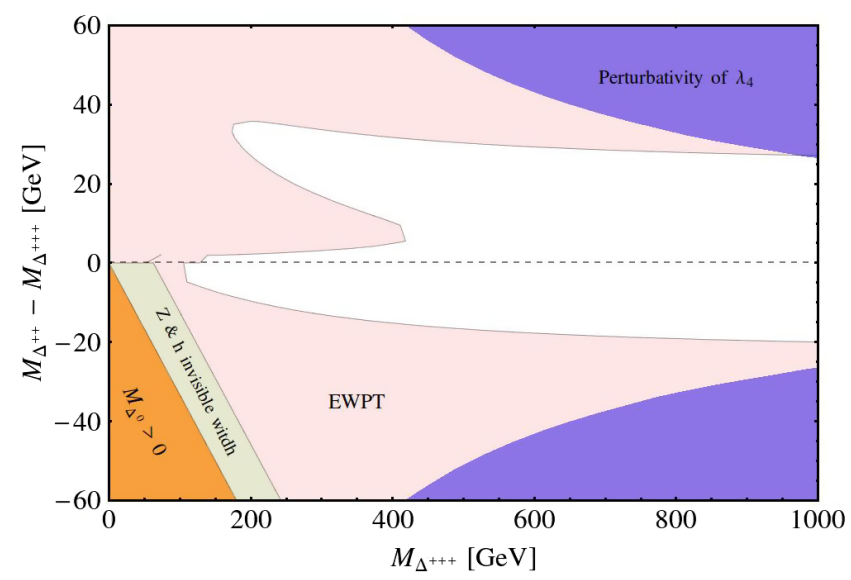

FIG. 4. Summary of few experimental and theoretical constraints in the $M_{\Delta^{++}}-M_{\Delta^{+++}}$parameter space. While the pink shade region is excluded by EWPT at 95\% C.L., the green region is ruled out by the measured $\mathrm{ZZ}$ and $h$ invisible widths. On the other hand, the blue and orange regions are excluded by the perturbativity of $\lambda_{4}(\leq \sqrt{4 \pi})$ and positivity of $M_{\Delta^{0}}$, respectively.

$Y^{\prime}$ to be unnaturally small $\left(\ll m_{e} / v_{H}\right)$ to obtain correct neutrino masses [11]. We deem $m_{e} / v_{H} \sim \mathcal{O}\left(10^{-6}\right)$ as a limit of natural Yukawa couplings since a smaller Yukawa coupling has not been observed in nature. So, in the spirit of the above argument, we work in a $v_{\Delta} \rightarrow 0$ paradigm in this section. In addition, the mixing terms between the SM-like Higgs $h \approx \operatorname{Re}\left(\phi^{0}\right)$ and $\operatorname{Re}\left(\Delta^{0}\right)$ are proportional to either $v_{\Delta}$ or $\lambda_{5}$. For $v_{\Delta} \ll v_{H}$, Eq. (2.4) tells us that we require $\left|\frac{\lambda_{5}}{v_{\Delta}}\right| \ll 1 \mathrm{GeV}^{-1}$ to achieve $\mathcal{O}(100-1000 \mathrm{GeV})$ mass for $\Delta$. Hence, applying the generic treatment of Ref. [30] is apt for our study.

The constraints on $S, T$, and $U$ are extracted from the global fit of the EW precision data. We use the fit results from the Gfitter Collaboration [31] for the reference SM parameters $m_{h}=126 \mathrm{GeV}$ and $m_{t}=173 \mathrm{GeV}$. The latest constraints are

$S_{\text {exp }}=0.03 \pm 0.10, \quad T_{\exp }=0.05 \pm 0.12$,

$U_{\text {exp }}=0.03 \pm 0.10$,

with relative correlations

$\rho_{S T}=0.89, \quad \rho_{T U}=-0.83, \quad \rho_{S U}=-0.54$.

Using the above experimental values, we constrain $M_{\Delta^{ \pm \pm \pm}}$ and $\lambda_{4}$ by means of a two parameter $\chi^{2}$ analysis. In Fig. 4, we show 95\% C.L. limits EWPT bounds on the $\Delta M-M_{\Delta^{ \pm \pm \pm}}$plane by the pink shaded region, with $\Delta M \equiv M_{\Delta^{ \pm \pm}}-M_{\Delta^{ \pm \pm \pm}} \approx \frac{\lambda_{4}}{8} \frac{v_{H}^{2}}{M_{\Delta^{ \pm \pm \pm}}}$. Additionally, we also present limits from perturbativity of $\lambda_{4}(\leq \sqrt{4 \pi})$ by the blue shaded region in Fig. 4. For large negative value of $\Delta M$, lighter members of the quadruplet will have negative masses. We constrain such scenarios by the orange shaded region. Also for $\Delta M<0$ scenarios neutral quadruplet members are the lightest, and $Z$ or $h$ bosons can decay to a pair of them. These neutral scalars then decay to neutrinos resulting in large invisible decay width of $Z$ and $h$ bosons measured at the LEP and LHC, respectively. The constraints on the above cases from the measured $Z$ and $h$ invisible decay widths are shown by the green shaded region in Fig. 4.

From Fig. 4, we can infer that at low $M_{\Delta^{ \pm \pm \pm}}$the bounds are dominated by the $S$ parameter. For larger $M_{\Delta^{ \pm \pm \pm}} \gtrsim 200 \mathrm{GeV}$, the limits form $T$ parameter take over, but for the very large value of $M_{\Delta^{ \pm \pm \pm}}>1 \mathrm{TeV}$, the perturbativity limit of $\lambda_{4}$ imposes the most stringent constraint on $\Delta M$. One important observation from the above figure is that EWPT limits the mass splitting of the quadruplets to be $\lesssim 30 \mathrm{GeV}$. This poses serious problems for collider searches of $\Delta^{ \pm \pm \pm}$(when it is the heaviest member of the quadruplet) or $\Delta^{ \pm \pm}$(all cases). For $\Delta M \gtrsim 10 \mathrm{GeV}$, cascade decay always dominates, and with $\Delta M \lesssim 30 \mathrm{GeV}$, the decay products will be too soft to pass LHC thresholds, as we shall demonstrate in Sec. V D.

\section{CONSTRAINTS FROM LFV EXPERIMENTS}

It is well known that experimental upper limits on leptonflavor-violating decays provide important constraints on $\mathrm{TeV}$-scale extensions of the standard model and thus it puts constraints on the free parameters of our model also. In the canonical SM seesaw, the LFV decay rates induced by the neutrino mixings are highly suppressed by the requirement that the scale of new physics is at $10^{15} \mathrm{GeV}$ and hence are well below the current experimental bounds. On the other hand, in the TeV scale BNT model, several new contributions appear due to the additional contributions from scalar quadruplet and triplet vectorlike lepton members, which could lead to sizeable LFV rates. Since we are concentrating on the scenario where vectorlike leptons $\Sigma^{\prime}$ s are heavy enough $(\sim 5 \mathrm{TeV})$, whereas scalar quadruplet members are as light as less than a $\mathrm{TeV}$, the contribution of vectorlike leptons ( $\Sigma^{\prime}$ s) to the lepton-flavor-violating process $\mu \rightarrow e \gamma$ is negligible compared to the contribution from the $\Delta$ members. We refer the reader to Ref. [15] for the
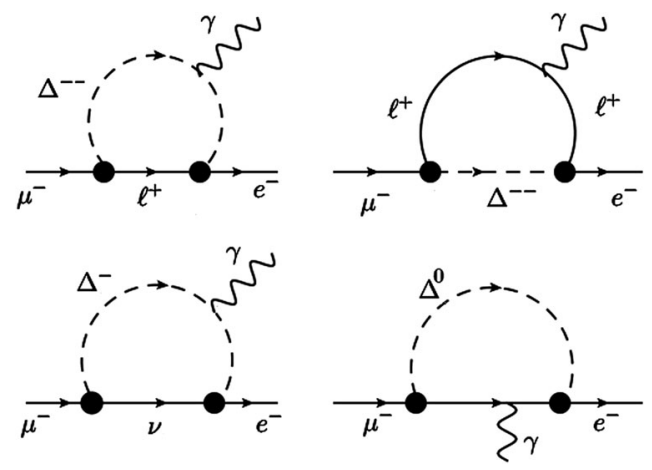

FIG. 5. Leading representative Feynman diagrams for the $\mu \rightarrow e \gamma$ process. 

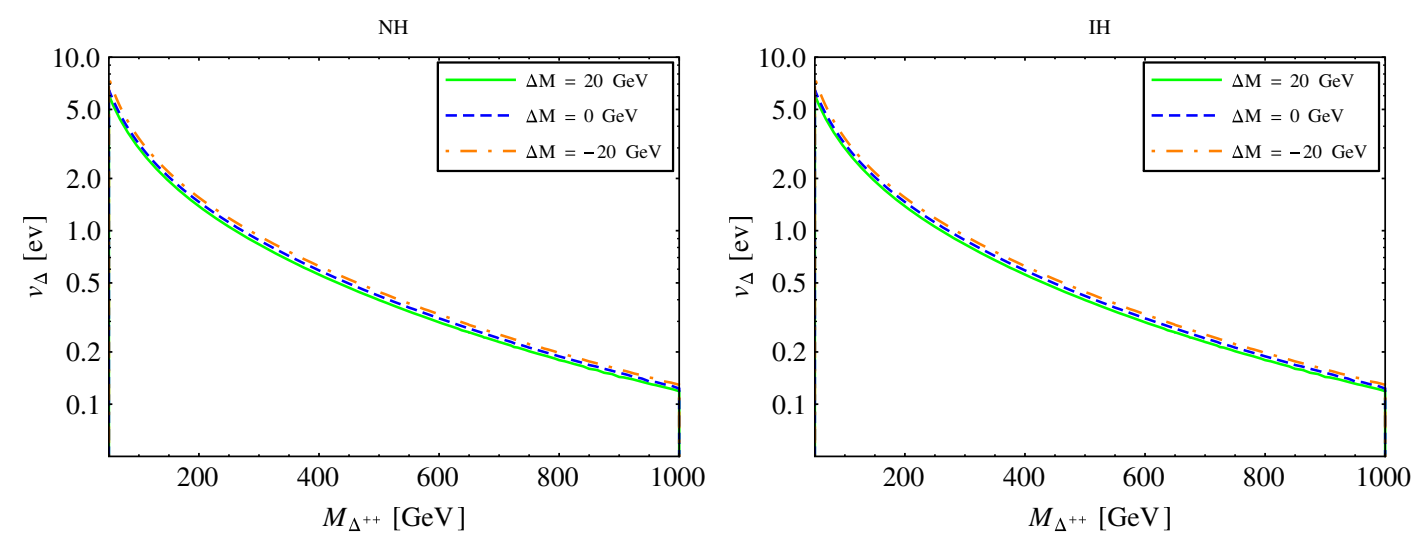

FIG. 6. Bounds on the $v_{\Delta}-M_{\Delta^{++}}$plane from lepton-flavor-violating $\mu \rightarrow e \gamma$ processes at 90\% C.L. for both $\mathrm{NH}$ (left) and IH (right) of neutrino masses. The area below the curves is ruled out.

complementary scenario. Leading representative Feynman diagrams for the $\mu \rightarrow e \gamma$ process are shown in Fig. 5. Here, charged scalars $\left(\Delta^{ \pm \pm}, \Delta^{ \pm}\right)$contribute more dominantly than the neutral one.

Then, LFV $\mu \rightarrow$ e $\gamma$ decay branching ratio can be easily calculated by

$$
B(\mu \rightarrow e \gamma)=\frac{\alpha_{\mathrm{QED}}\left|\left(M_{\nu}^{2}\right)_{e \mu}\right|^{2}}{108 \pi G_{F}^{2} D^{4}}\left[\frac{1}{M_{\Delta^{++}}^{2}}+\frac{1}{4 M_{\Delta^{+}}^{2}}\right]^{2},
$$

where $M_{\nu}=\left(m_{\nu}\right)^{\text {tot }}$ and $D$ is defined in Eq. (5.10).

We have used the currently most stringent bound by the MEG Collaboration, $\operatorname{BR}(\mu \rightarrow e \gamma)<\left(5.7 \times 10^{-13}\right)$ at $90 \%$ C.L. [16], and the bound on VEV $v_{\Delta}$ as a function of $M_{\Delta^{++}}$for a given mass splitting of the charged scalars is shown in Fig. 6 for both NH (left) and IH (right). The region below the respective lines is ruled out, and $\mu \rightarrow e \gamma$ essentially provides a lower bound on $v_{\Delta}$. As we can see from Eq. (4.1), the contribution from the doubly charged Higgs is the most dominant one. Mass splitting between $\Delta$ members has no significant impact in $\mu \rightarrow e \gamma$ limits. Also, the above limits are not sensitive to the mass ordering of neutrinos. However, in this model, there exists a tree-level diagram for $\mu \rightarrow 3 e$ mediated by the doubly charged scalar. It is worth mentioning that the constraints from $\mu \rightarrow 3 e$ are less stringent [32] than the corresponding limits arising from the $\mu \rightarrow$ er process. We do not explicitly discuss here other LFV processes, such as $\mu \rightarrow e$ conversion in nuclei or electric dipole moments [33], which are left for future studies for detail since they also impose weaker bounds on our parameter space compared to $\mu \rightarrow e \gamma$.

\section{COLLIDER IMPLICATIONS}

This model provides an interesting avenue to test the neutrino mass generation mechanism at the LHC. The presence of the isospin $3 / 2$ scalar multiplet can give rise to rich phenomenology at the LHC. The collider signatures of the BNT model have been studied in the literature $[11,12]$.
However, there has not only been new data made public by the LHC experiments since then, resulting in updated constraints, but also a few subtle points regarding the phenomenology of multicharged Higgs particles that were absent in previous analyses need to be clarified. In this section, we try to investigate the limits on the $\Delta$ masses from the recent experimental data.

\section{A. Constraints from $h \rightarrow \gamma \gamma$ at the LHC}

The BNT model is rich in multicharged scalars. These multicharged scalars can mediate SM-like Higgs decay to a pair of photons in addition to $t$ and $W$ loops. A representative triangle loop diagram for these processes is shown in Fig. 7. In fact, the $\Delta$ mediated processes can either augment or suppress the SM predicted $h \rightarrow \gamma \gamma$ rate at the LHC depending on the signs and relative strengths of $\lambda_{3}$ and $\lambda_{4}$. This is because the couplings between the SM-like Higgs $h$ and a pair of singly, doubly, and triply charged Higgs are

$$
\begin{aligned}
& \tilde{\lambda}_{1}=v_{H}\left(\lambda_{3}+\frac{\lambda_{4}}{4}\right) \\
& \tilde{\lambda}_{2}=v_{H}\left(\lambda_{3}-\frac{\lambda_{4}}{4}\right) \\
& \tilde{\lambda}_{3}=v_{H}\left(\lambda_{3}-\frac{3 \lambda_{4}}{4}\right),
\end{aligned}
$$

respectively.

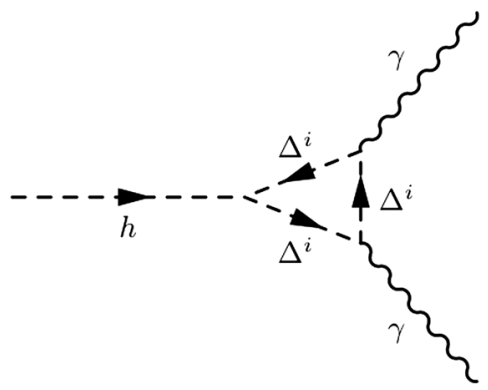

FIG. 7. Triangle diagrams that mediate $h \rightarrow \gamma \gamma$ decay in the BNT model. Here, $\Delta^{i}$ stands for singly, doubly, and triply charged Higgs. 
For a given production process of a Higgs, denoted by $X$, and the subsequent decay into final state $Y$, the signal strength parameter, normalized to the SM values, is defined as

$$
\mu_{Y}=\frac{\sigma_{X}}{\sigma_{X}^{\mathrm{SM}}} \frac{\Gamma_{h \rightarrow Y}}{\Gamma_{h \rightarrow Y}^{\mathrm{SM}}} \frac{\Gamma_{h, \mathrm{tot}}^{\mathrm{SM}}}{\Gamma_{h, \mathrm{tot}}} .
$$

In our study, the new physics can influence only the total decay width, $\Gamma_{h, \text { tot }}$, and the partial decay rate, $\Gamma_{h \rightarrow Y}$. We formulate this change in the $h \gamma \gamma$ coupling as

$$
g_{h \gamma \gamma}=\kappa_{\gamma} g_{h \gamma \gamma}^{\mathrm{SM}}
$$

where [34-36]

$\kappa_{\gamma}=\frac{\left|\frac{N_{t}^{c} Q_{t}^{2}}{v_{H}} A_{\frac{1}{2}}\left(\tau_{t}\right)+\frac{1}{v_{H}} A_{1}\left(\tau_{W}\right)+\sum_{i=1}^{3} \frac{\tilde{\lambda}_{i} Q_{i}^{2}}{2 M_{i}} A_{0}\left(\tau_{i}\right)\right|^{2}}{\left|\frac{N_{t}^{c} Q_{t}^{2}}{v_{H}} A_{\frac{1}{2}}\left(\tau_{t}\right)+\frac{1}{v_{H}} A_{1}\left(\tau_{W}\right)\right|^{2}}$.

Here, the loop functions are given by [34]

$$
\begin{aligned}
A_{0} & =-\tau+\tau^{2} f(\tau), \\
A_{\frac{1}{2}}(\tau) & =2 \tau[1+(1-\tau) f(\tau)], \\
A_{1} & =-2-3 \tau(1+(2-\tau) f(\tau)),
\end{aligned}
$$

with

$$
f(x)= \begin{cases}\arcsin ^{2}[1 / \sqrt{x}], & \text { if } x \geq 1 \\ -\frac{1}{4}\left[\ln \frac{1+\sqrt{1-x}}{1-\sqrt{1-x}}-i \pi\right]^{2}, & \text { if } x<1\end{cases}
$$

The parameters $\tau_{i}=4 M_{i}^{2} / M_{h}^{2}$ are defined by the corresponding masses of the heavy loop particles. Thus, the partial decay width of the SM-like Higgs to $\gamma \gamma$ can be written as

$$
\frac{\Gamma_{h \rightarrow \gamma \gamma}}{\Gamma_{h \rightarrow \gamma \gamma}^{\mathrm{SM}}}=\kappa_{\gamma}^{2} .
$$

Consequently, the total decay width of $h$ in terms of the rescaling factor $\kappa_{\gamma}$ is $[37,38]$

$$
\frac{\Gamma_{h, \text { tot }}}{\Gamma_{h, \text { tot }}^{\mathrm{SM}}} \approx 0.9977+0.0023 \kappa_{\gamma}^{2},
$$

with $\Gamma_{h \text { tot }}^{\mathrm{SM}}=4.07 \mathrm{MeV}$ [37].

CMS and ATLAS both recently made public their $h \rightarrow \gamma \gamma$ analysis, combining all production channels, based on $\sim 36 \mathrm{fb}^{-1}$ of data at $13 \mathrm{TeV}$ center-of-mass energy. The measured strengths $\left(\mu_{\gamma}\right)$ of the above decay rate by CMS [39] and ATLAS [40] are $\mu_{\gamma}^{\mathrm{CMS}}=1.16_{-0.14}^{+0.15}$ and $\mu_{\gamma}^{\text {ATLAS }}=0.99 \pm 0.14$, respectively. In Fig. 8, we overlay the limits obtained from $\mu_{\gamma}^{\mathrm{CMS}}$, shown by brown shaded regions, on top of EWPT excluded regions in the $\Delta M-$ $M_{\Delta^{ \pm \pm \pm}}$plane. From Eq. (5.2), we can notice that the strength of $\mu_{\gamma}$ in the BNT model is controlled by a combination of $\lambda_{3}$ and $\lambda_{4}$. In the results of Fig. $8, \lambda_{4}$ is fixed by $\Delta M$. So, we show our results in the above figure for four values of $\lambda_{3}= \pm 1, \pm 0.1$. In Fig. 9, we plot the same bounds from $\mu_{\gamma}^{\text {ATLAS }}$. The shapes of exclusion contours from CMS and ATLAS differ marginally for the same value of $\lambda_{3}$ since the measured $\mu_{\gamma}$ by them are not the same.

We notice from Figs. 8 and 9 that $h \rightarrow \gamma \gamma$ limits depend strongly on the magnitude of $\lambda_{3}$. For $\left|\lambda_{3}\right| \gtrsim 1, h \rightarrow \gamma \gamma$ excludes a relatively large fraction of the parameter space that is not ruled out by EWPT. In contrast, if $\left|\lambda_{3}\right|$ assumes a small value $(\lesssim 0.1)$, it will hardly add anything on top of EWPT bounds.

\section{B. Production of $\boldsymbol{\Delta}^{ \pm \pm}$and $\boldsymbol{\Delta}^{ \pm \pm \pm}$at the LHC}

A pair of $\Delta^{ \pm \pm \pm}\left(\Delta^{ \pm \pm}\right)$can be produced at the LHC by the Drell-Yan (DY) process via $s$-channel $\gamma^{*} / Z$ boson exchange. Also, associated production of $\Delta^{ \pm \pm \pm} \Delta^{\mp \mp}\left(\Delta^{ \pm \pm} \Delta^{\mp}\right)$ is possible via $s$-channel $W$ exchange. The relevant diagrams for such processes are shown in Fig. 10. Being $s$ channel, DY pair production cross sections are significantly suppressed for large $\Delta^{ \pm \pm \pm}\left(\Delta^{ \pm \pm}\right)$masses. Additionally, due to large electromagnetic charges carried by $\Delta^{ \pm \pm \pm}\left(\Delta^{ \pm \pm}\right)$, they can be pair produced by photon fusion (PF) as well. We refer the reader to Ref. [12] for Feynman diagrams relevant for the above process. In comparison with DY, photoproduction of these multicharged scalars takes place via $t$ - and $u$-channel processes mediated by charged scalars and hence falls less sharply for higher $\Delta$ masses. Although the photoproduction cross section of triply and doubly charged scalars benefits from enhancements by factors of $3^{4}$ and $2^{4}$, respectively, due to their large electric charges, it is suppressed, at the same time, by the tiny parton density of a photon inside a proton. For a detailed discussion on the parton density function of photons from different collaborations, we refer the reader to Refs. [12,41]. In this study, we use the NNPDF23_LO_AS_130 parton distribution function (PDF) set [42], which contains a photon PDF. It is important to point out that, although including PF boosts the production cross section for heavier masses, $P F$ processes also suffer from large uncertainties. In this analysis, we build on the work of the above references and include the errors associated with using all the available eigenvector sets of a given PDF.

In Fig. 11, we present cross sections of various pair production and associated production processes. We employ the MADGRAPH5_AMC@NLO_V2.5.4 code [43] for our calculation, where the BNT model is implemented using FeynRules_v2.0 [44]. We have not used any $K$ factor in the above computations. Pair production of $\Delta^{ \pm \pm \pm}$ and $\Delta^{ \pm \pm}$via the DY mechanism is shown by green and thin orange lines, respectively. The same for the above two particles in a combination of DY and PF is depicted by 

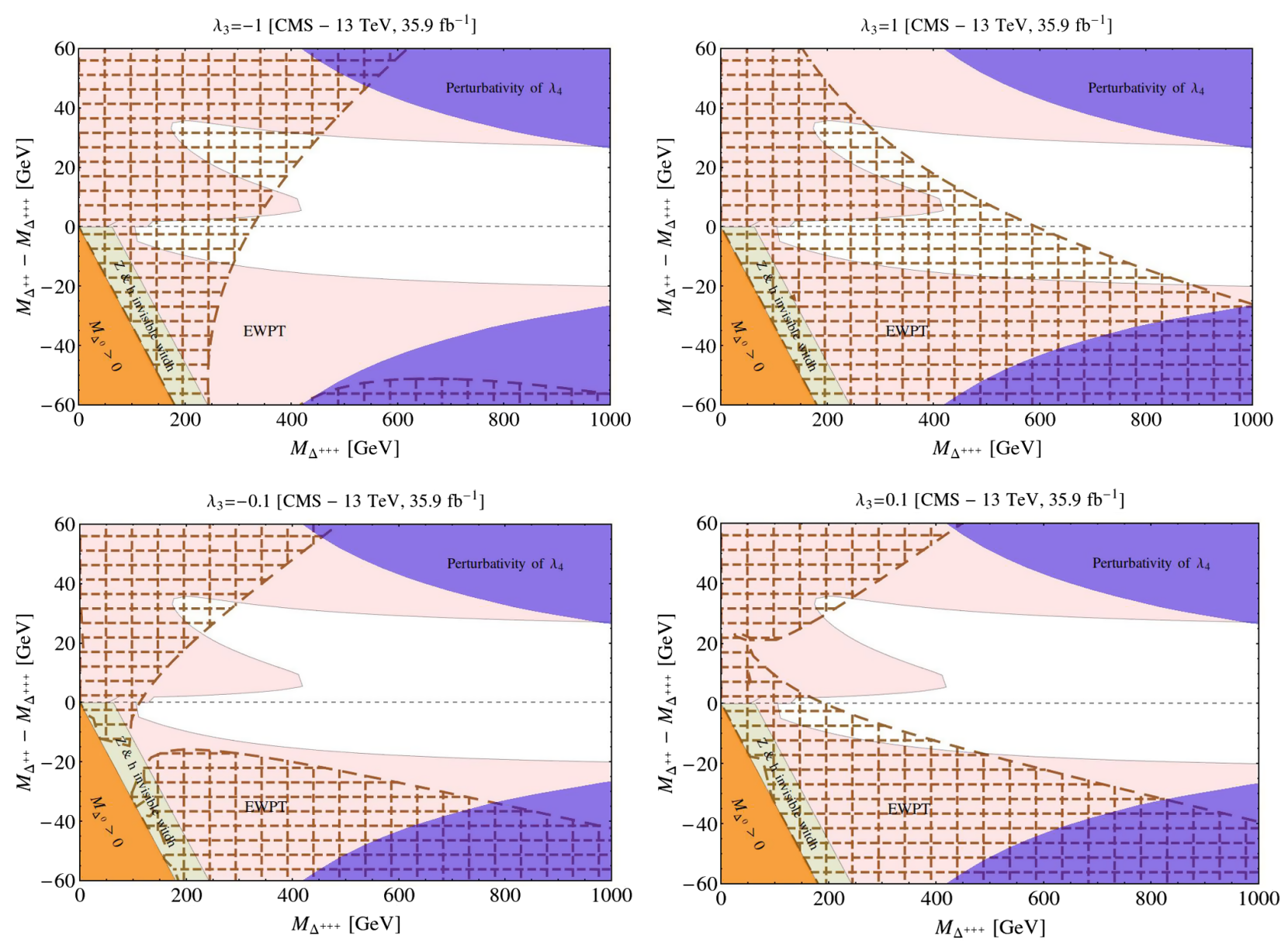

FIG. 8. Constraints from $h \rightarrow \gamma \gamma$ decay rate measured by CMS in the $M_{\Delta}-M_{\Delta^{ \pm \pm \pm}}$plane are shown by the brown shaded region. We plot the limits for $\lambda_{3}=-1$ (top left), 1 (top right), -0.1 (bottom left), and 1 (bottom right). The other colored regions have the same meaning as Fig. 4.

dot-dashed red and dashed blue lines. In contrast, dashed brown and thick yellow lines represent associated production cross sections for the same two particles. The uncertainties related to each process due to PDF variation are encoded within a band of the same color as the respective cross section curve. As expected, the presence of $t$-channel diagrams of PF enhances pair production cross sections of both doubly and triply charged bosons significantly for masses above $500 \mathrm{GeV}$. However, while errors of DY processes are tiny ( $\sim 5 \%)$, the large error bands of the two channels that include $\mathrm{PF}$ will not escape the reader's attention. In fact, the error of $\mathrm{DY}+\mathrm{PF}$ channels is $>100 \%$ for $M_{\Delta} \gtrsim 500 \mathrm{GeV}$. So, one can infer from the results of Fig. 11 that, although adding PF to DY production provides an apparent enhancement in the pair production cross section, one cannot be certain about such an increase in the cross section due to the enormous PDF uncertainty associated with PF. Hence, we ignore the inclusion of PF in this paper.

\section{Decay of $\Delta^{ \pm \pm}$and $\Delta^{ \pm \pm \pm}$}

In this section, we discuss the decay of doubly and triply charged Higgs bosons of the BNT model in details.
Especially, we shall pay particular attention to the proper decay length of these particles and the corresponding implications for their LHC detection. Another point we want to emphasize is that for our choice of $M_{\Sigma}=5 \mathrm{TeV}$, $\left(m_{\nu}\right)_{i j}^{\text {tree }} \sim\left(m_{\nu}\right)_{i j}^{\text {loop }}$ for a range of $M_{\Delta}$ that is accessible to the future high luminosity LHC run. The interplay between these two contributions should reflect in the leptonic BRs of the quadruplet components. This point was not considered by previous LHC studies [11,12] of the BNT model. The inclusion of the dimension-5 loop contribution to the Yukawa couplings changes the value of $v_{\Delta}$ where the crossover from leptonic to bosonic decay channels takes place.

First, let us quantify the impact of the inclusion of the dimension-5 contribution to the Yukawa couplings. In the absence of the dimension-5 operator, from Eqs. (2.11) and (2.12), one can deduce the Feynman rule corresponding to the coupling of lepton doublets with the Higgs quadruplet $-\frac{2}{\sqrt{3}} \frac{\left(Y_{i} Y_{j}^{\prime}+Y_{j} Y_{i}^{\prime}\right) v_{H}}{\sqrt{2} M_{\Sigma}}=\frac{2}{\sqrt{6}} \frac{\left(m_{\nu}\right)_{i j}^{\text {tree }}}{v_{\Delta}}$, where the prefactor 2 in the numerator arises since the coupling can come from two vertices and the other factor $1 / \sqrt{3}$ comes from the 

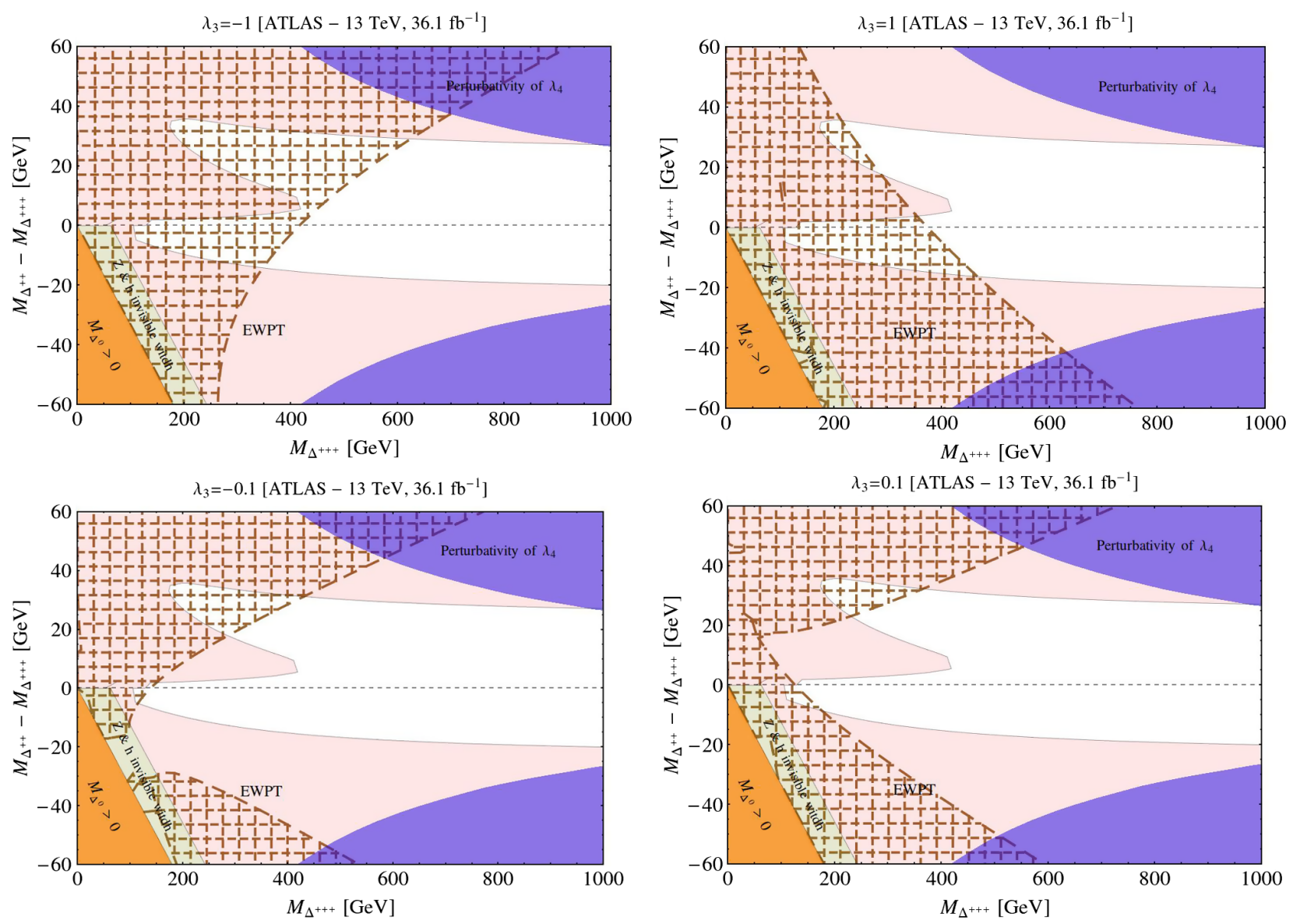

FIG. 9. Constraints from $h \rightarrow \gamma \gamma$ decay rate measured by ATLAS in the $M_{\Delta}-M_{\Delta^{ \pm \pm \pm}}$plane are shown by the brown shaded region. We plot the limits for $\lambda_{3}=-1$ (top left), 1 (top right), -0.1 (bottom left), and 1 (bottom right). The other colored regions have the same meaning as Fig. 4.

Clebsch-Gordon coefficient related to the interaction of Eq. (2.11), as described in Appendix A. Now, if we include the loop contribution, the above Feynman rule is modified to

$$
h_{i j}=-\frac{2}{\sqrt{3}} \frac{\left(Y_{i} Y_{j}^{\prime}+Y_{j} Y_{i}^{\prime}\right) v_{H}}{\sqrt{2} M_{\Sigma}}=\frac{2}{\sqrt{6}} \frac{\left(m_{\nu}\right)_{i j}^{\mathrm{tot}}}{D},
$$

where $\left(m_{\nu}\right)_{i j}^{\text {tot }}=\left(m_{\nu}\right)_{i j}^{\text {tree }}+\left(m_{\nu}\right)_{i j}^{\text {loop }}$ and $D$ is given by

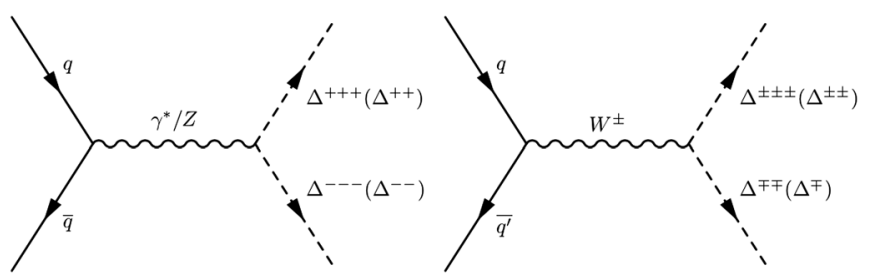

FIG. 10. Pair production (left) and associated production (right) of $\Delta^{ \pm \pm \pm}\left(\Delta^{ \pm \pm}\right)$via DY processes.

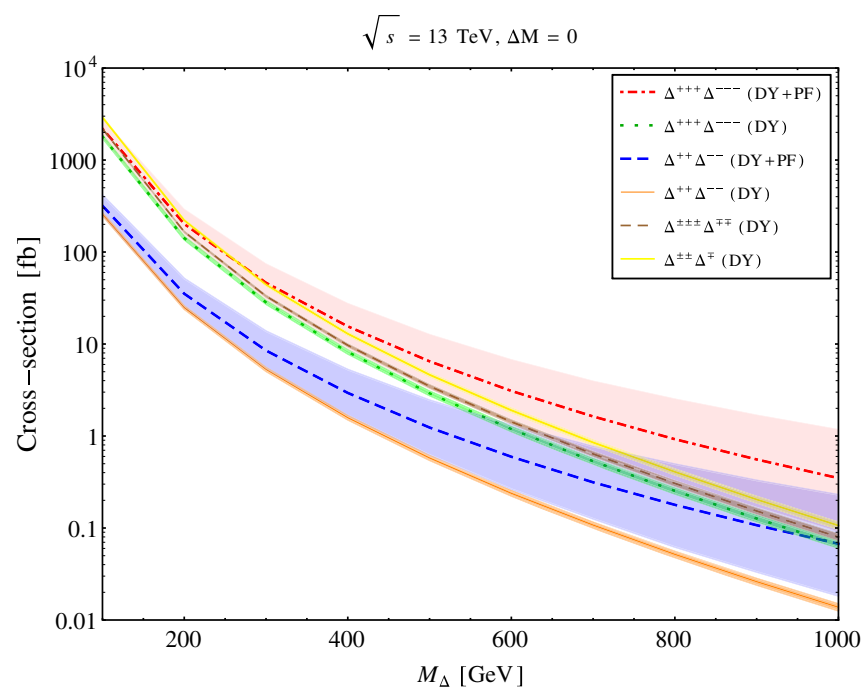

FIG. 11. The cross sections of various pair production and associated production channels for $\sqrt{s}=13 \mathrm{TeV}$. No mass splitting between the quadruplet components is considered here. Large mass splittings will change associated production cross sections. The uncertainties associated with the variation of PDF eigenvector sets are shown by bands of the same color as the cross section curves. 


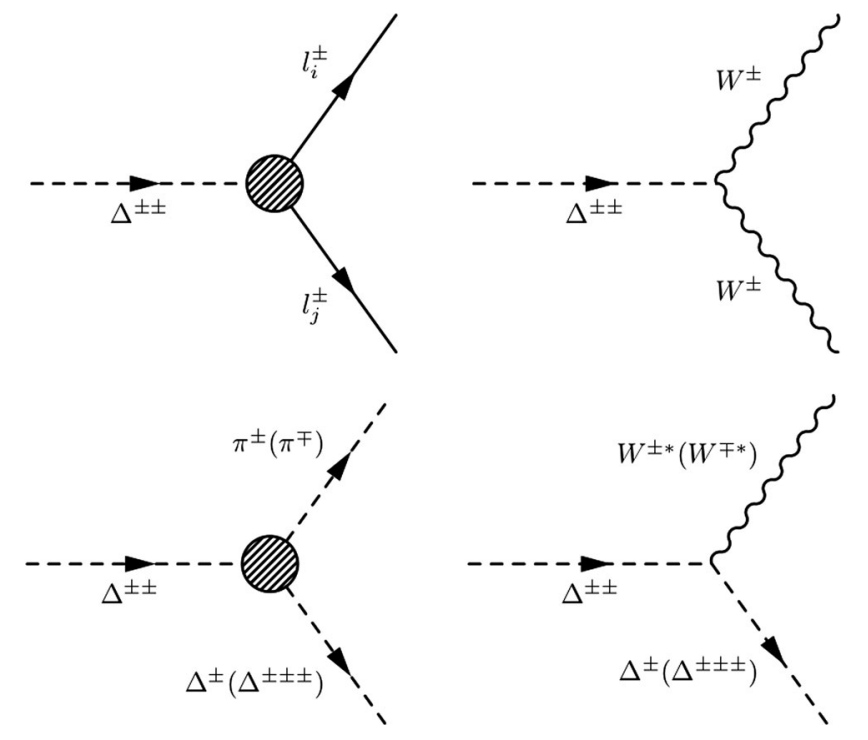

FIG. 12. Feynman diagrams for decay of $\Delta^{ \pm \pm}$.

$$
\begin{aligned}
D= & v_{\Delta}-\frac{(3+\sqrt{3}) \lambda_{5} v_{H} M_{\Sigma}^{2}\left(Y_{i} Y_{j}^{\prime}+Y_{i}^{\prime} Y_{j}\right)}{32 \pi^{2}\left(M_{\Delta}^{2}-M_{H}^{2}\right)} \\
& \times\left(\frac{M_{\Delta}^{2} \log \left(\frac{M_{\Sigma}^{2}}{M_{\Delta}^{2}}\right)}{M_{\Sigma}^{2}-M_{\Delta}^{2}}-\frac{M_{H}^{2} \log \left(\frac{M_{\Sigma}^{2}}{M_{H}^{2}}\right)}{M_{\Sigma}^{2}-M_{H}^{2}}\right) .
\end{aligned}
$$

Next, we list the decay widths of doubly charged Higgs in various channels. The corresponding Feynman diagrams are shown in Fig. 12. The decay of $\Delta^{ \pm \pm}$can happen in four possible channels. While $l_{i}^{ \pm} l_{j}^{ \pm}$and $W^{ \pm} W^{ \pm}$final states are always accessible, cascade decays $\Delta \pi$ and $\Delta W^{*}$ open up only when the mass splitting between quadruplet members is nonzero. We should note that $\Delta^{ \pm \pm}$cannot be the lightest or heaviest member of the $\Delta$ multiplet under any circumstances. Hence, for the nonzero mass gap, it can decay in cascades via $\Delta^{ \pm} X^{ \pm}$or $\Delta^{ \pm \pm \pm} X^{\mp}$ (where $X=\pi, W^{*}$ ) depending on whether $\Delta M<0$ or $\Delta M>0$. So, the relevant decay width formulas of $\Delta^{ \pm \pm}$are $[45,46]$

$$
\begin{aligned}
\Gamma\left(\Delta^{ \pm \pm} \rightarrow l_{i}^{ \pm} l_{j}^{ \pm}\right) & =\frac{\left|h_{i j}\right|^{2} M_{\Delta^{ \pm \pm}}}{4 \pi\left(1+\delta_{i j}\right)}\left(1-\frac{m_{i}^{2}}{M_{\Delta^{ \pm \pm}}^{2}}-\frac{m_{j}^{2}}{M_{\Delta^{ \pm \pm}}^{2}}\right)\left[\lambda\left(\frac{m_{i}^{2}}{M_{\Delta^{ \pm \pm}}^{2}}, \frac{m_{j}^{2}}{M_{\Delta^{ \pm \pm}}^{2}}\right)\right]^{1 / 2}, \\
\Gamma\left(\Delta^{ \pm \pm} \rightarrow W^{ \pm} W^{ \pm}\right) & =S_{W^{ \pm} W^{ \pm}}^{2} \frac{g^{4} v_{\Delta^{2}}^{2} M_{\Delta^{ \pm \pm}}^{3}}{16 \pi M_{W}^{4}}\left(\frac{3 M_{W}^{4}}{M_{\Delta^{ \pm \pm}}^{4}} \frac{M_{W}^{2}}{M_{\Delta^{ \pm \pm}}^{2}}+\frac{1}{4}\right) \beta\left(\frac{M_{W}^{2}}{M_{\Delta^{ \pm \pm}}^{2}}\right), \\
\Gamma\left(\Delta^{ \pm \pm} \rightarrow \Delta^{ \pm} \pi^{ \pm}\right) & =S_{\Delta^{ \pm} W^{ \pm}}^{2} \frac{g^{4}\left|V_{u d}\right|^{2} \Delta M^{3} f_{\pi}^{2}}{16 \pi M_{W}^{4}} \\
\Gamma\left(\Delta^{ \pm \pm} \rightarrow \Delta^{ \pm} l^{ \pm} \nu_{l}\right) & =S_{\Delta^{ \pm} W^{ \pm}}^{2} \frac{g^{4} \Delta M^{5}}{240 \pi^{3} M_{W}^{4}}, \\
\Gamma\left(\Delta^{ \pm \pm} \rightarrow \Delta^{ \pm} q \overline{q^{\prime}}\right) & =3 \Gamma\left(\Delta^{ \pm \pm} \rightarrow \Delta^{ \pm} l^{ \pm} \nu_{l}\right), \\
\Gamma\left(\Delta^{ \pm \pm} \rightarrow W^{ \pm} W^{ \pm *}\right) & =S_{W^{ \pm} W^{ \pm}}^{2} \frac{3 g^{6} M_{\Delta^{ \pm \pm}}}{512 \pi^{3}} \frac{v_{\Delta}^{2}}{M_{W}^{2}} F\left(\frac{M_{W}^{2}}{M_{\Delta^{ \pm \pm}}^{2}}\right),
\end{aligned}
$$

where $S_{W^{ \pm} W^{ \pm}}=\sqrt{3}$ and $S_{\Delta^{ \pm} W^{ \pm}}=\sqrt{2}$ are scale factors that we use to convert the expressions of decay widths given in Refs. [45,46] for the $S U(2)$ triplet to the quadruplet. Here, $V_{u d}$ is the $u d$ element of the Cabibbo-Kobayashi-Maskawa matrix, and $f_{\pi}=131 \mathrm{MeV}$ is the pion decay constant. One can easily use the results of $\Gamma\left(\Delta^{ \pm \pm} \rightarrow \Delta^{ \pm} X^{ \pm}\right)$to derive $\Gamma\left(\Delta^{ \pm \pm} \rightarrow\right.$ $\left.\Delta^{ \pm \pm \pm} X^{\mp}\right)$ decay widths by changing the scale factor from $S_{\Delta^{ \pm} W^{ \pm}}$to $S_{\Delta^{ \pm \pm \pm} W^{\mp}}=\sqrt{3 / 2}$. The kinematic functions are given by

$$
\begin{aligned}
\lambda(x, y) & =1+x^{2}+y^{2}-2 x y-2 x-2 z, \\
\beta(x) & =\sqrt{1-4 x} \\
F(x) & =-|1-x|\left(\frac{47}{2} x-\frac{13}{2}+\frac{1}{x}\right)+3\left(1-6 x+4 x^{2}\right)|\log \sqrt{x}|+\frac{3\left(1-8 x+20 x^{2}\right)}{\sqrt{4 x-1}} \arccos \left(\frac{3 x-1}{2 x^{3 / 2}}\right) .
\end{aligned}
$$

In Fig. 13, we present a set of representative decay phase diagrams of $\Delta^{ \pm \pm}$in the $\Delta M-v_{\Delta}$ plane for $M_{\Delta^{ \pm \pm}}=$ $400 \mathrm{GeV}$. In the top panel, we show the scenarios when $\Delta M<0$ (left) and $\Delta M>0$ (right), respectively, for $\mathrm{NH}$ of neutrino masses. In the lower panel, the same is shown for IH. The feature of four plots is almost identical. From Eq. (5.11), it is clear that the leptonic decay BR of $\Delta^{ \pm \pm}$falls with $v_{\Delta}$ but the gauge boson decay $\mathrm{BR}$ increases with $v_{\Delta}$.
The crossover between the leptonic decay dominated region to the gauge boson dominated one happens at $v_{\Delta}=$ $4.6 \times 10^{-5}\left(5.4 \times 10^{-5}\right) \mathrm{GeV}$ for $\mathrm{NH}$ (IH) with $\Delta M \sim 0$. Neglecting the loop contribution in the leptonic couplings of Eq. (5.9) will shift the crossover point to a $18 \%$ higher value in $v_{\Delta}$ for both $\mathrm{NH}$ and IH. On the other hand, cascade decay channels open up for $\Delta M \neq 0$, and they become dominant for $\Delta M \approx 2-20 \mathrm{GeV}$ depending on the exact 

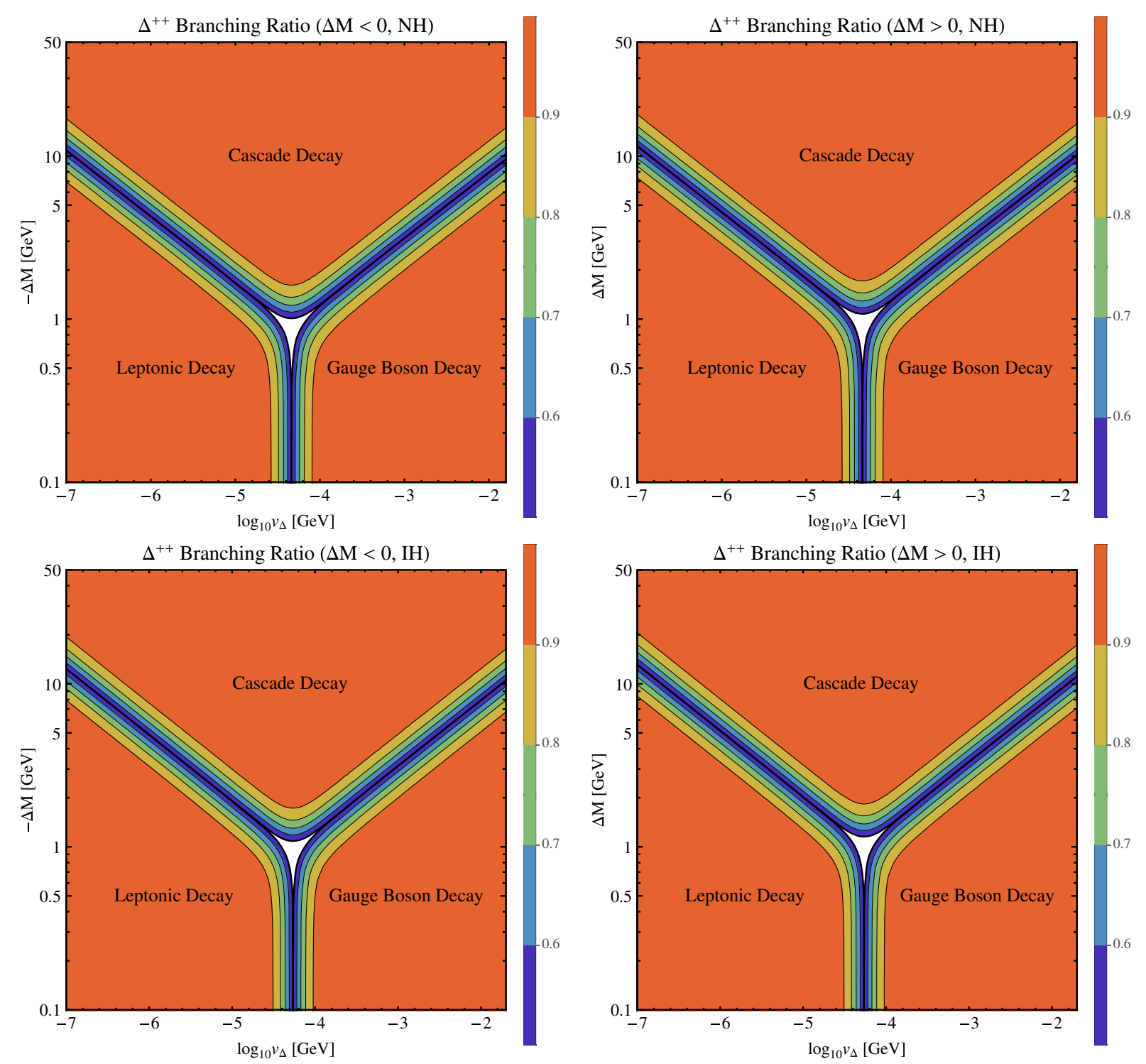

FIG. 13. Generic decay phase diagram for $\Delta^{ \pm \pm}$decays in the BNT model, with $M_{\Delta^{ \pm \pm}}=400 \mathrm{GeV}$. In the top panel, we show the scenarios when $\Delta M<0$ (left) and $\Delta M>0$ (right), respectively, for NH of neutrino masses. In the lower panel, the same is shown for IH. Here, $\Delta M=M_{\Delta^{ \pm \pm}}-M_{\Delta^{ \pm \pm \pm}}$.

value of $v_{\Delta}$. Now, a few comments are in order for cascade decay channels. Clearly, for $\Delta M$ below the charged pion mass of $140 \mathrm{MeV}$, the only cascade decay channels open are $\Delta^{ \pm \pm} \rightarrow \Delta^{ \pm} l^{ \pm} \nu_{l}(l=e, \mu)$. Once the pion channel is open, it will dwarf the leptonic channels decay width. Then, at their respective masses, other charged mesons like kaon channels will be accessible. However, they will always be subdominant compared to the pion channel. For $\Delta M>m_{\tau}$, the third lepton channel will be available. Finally, for $\Delta M \sim \mathcal{O}(2 \mathrm{GeV})$, the light quarks will cease to be confined, and they can be treated as free particles. So, at this stage, we can ignore the mesonic decay channels and replace them by $\Delta^{ \pm \pm} \rightarrow \Delta^{ \pm} q \overline{q^{\prime}}$.

Let us focus now on the total decay width $\Delta^{ \pm \pm}$. We have seen above that the total decay width of $\Delta^{ \pm \pm}$depends on neutrino and Higgs quadruplet parameters. In Fig. 14, we present the proper decay length, $c \tau$, of $\Delta^{ \pm \pm}$for four different settings of $M_{\Delta^{ \pm \pm}}$and $\Delta M$ for both $\mathrm{NH}$ (left panel) and IH (right panel). As seen in Fig. 14, $c \tau \gtrsim 10 \mu \mathrm{m}$ is achievable for $M_{\Delta^{ \pm \pm}} \lesssim 200 \mathrm{GeV}$. A general feature of both plots of the above figure is that the proper decay length is maximum when the crossover between $l l$ and $W W$ dominant regions happens at $v_{\Delta} \sim 10^{-5}-10^{-4} \mathrm{GeV}$ with $\Delta M=0$. However, the introduction of even a tiny mass splitting reduces $c \tau$ drastically since the cascade decay channels start dominating. Cascade decay widths are not tiny since they are not proportional to small parameter $v_{\Delta}$ or $m_{\nu}$. In Fig. 14, we show a few cases for $\Delta M= \pm 2.5 \mathrm{GeV}$ to illustrate this behavior. Given the total decay width of $\Delta^{ \pm \pm}$we obtained, it cannot be a long-lived charged 

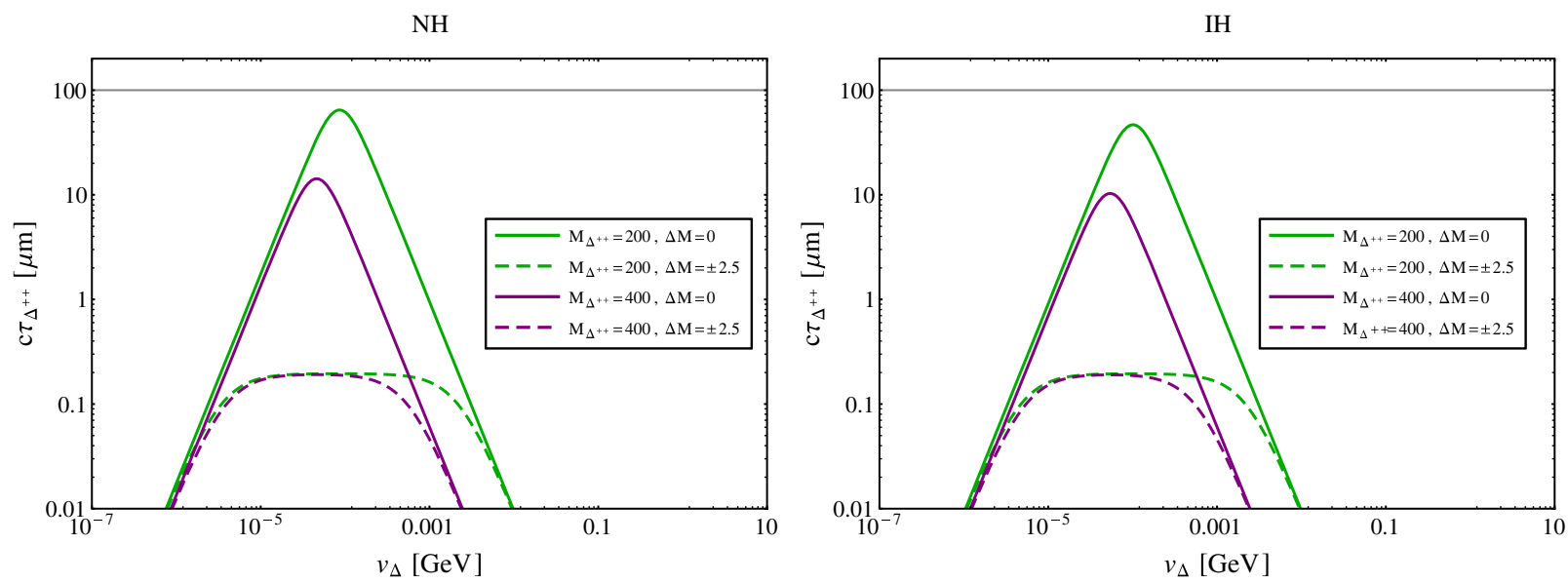

FIG. 14. Proper decay length of $\Delta^{ \pm \pm}$for different values of $M_{\Delta^{ \pm \pm}}$and $\Delta M$ for both NH (left) and IH (right) of neutrino masses. The gray horizontal lines in both panels refer to the limiting value of $c \tau$, up to which prompt-lepton searches at the LHC remain sensitive.

particle, but they can possibly give rise to large displaced vertices. To place our calculated $c \tau_{\Delta^{ \pm \pm}}$in some perspective, we want to draw the reader's attention to the latest CMS search of $\Delta^{ \pm \pm}$[47]. This prompt lepton study is sensitive to lepton tracks that start from a distance of $\mathcal{O}(100 \mu \mathrm{m})$ from the primary vertex (see Sec. IV of Ref. [47]). Also, the CMS Collaboration initiates its displaced vertex searches for a proper decay length of $\mathcal{O}(100 \mu \mathrm{m})$ [48]. We highlight this threshold proper decay length value by gray horizontal lines in Figs. 14 and 17. Hence, when BRs of $\Delta^{ \pm \pm}$in $l l$ and $W W$ channels are comparable, it may remain beyond the traditional prompt-lepton searches of the LHC for a small range of $\Delta^{ \pm \pm}$mass $(\lesssim 200 \mathrm{GeV})$ with $\Delta M \sim 0$.

Finally, we investigate various decay channels of the triply charged Higgs. In the BNT model, $\Delta^{ \pm \pm \pm}$can be the lightest (heaviest) particle of the quadruplet for the $\Delta M>$ $0(\Delta M<0)$ case. In the first case, it can only decay in three-body final states $l l W$ or $W W W$ via an off-shell $\Delta^{ \pm \pm}$ exchange. In the latter case, it will always decay to either $\Delta^{ \pm \pm} W^{ \pm *}$ or $\Delta^{ \pm \pm} \pi^{ \pm}$. The relevant Feynman diagrams are presented in Fig. 15. Decay of $\Delta^{ \pm \pm \pm}$, when it is the lightest, is a unique feature of this model. We discuss these decay channels in detail below. On the other hand, for $\Delta M<0$, the decay of $\Delta^{ \pm \pm \pm}$is very similar to $\Delta^{ \pm \pm}$decay, and one can easily convert the results of Eq. (5.11) for this purpose. The decay widths of $\Delta^{ \pm \pm \pm}$for the $\Delta M \geq 0$ scenarios are given by

$$
\begin{aligned}
\Gamma\left(\Delta^{ \pm \pm \pm} \rightarrow l_{i}^{ \pm} l_{j}^{ \pm} W^{ \pm}\right) & =\frac{g^{2}}{1536\left(1+\delta_{i j}\right) \pi^{3}} \frac{M_{\Delta^{ \pm \pm \pm}}\left(m_{\nu}\right)_{i j}^{\text {tot2 }}}{v_{\Delta}^{2}} J \\
\Gamma\left(\Delta^{ \pm \pm} \rightarrow W^{ \pm} W^{ \pm} W^{ \pm}\right) & =\frac{3 g^{6}}{4096 \pi^{3}} \frac{M_{\Delta^{ \pm \pm \pm}}^{5} v_{\Delta}^{2}}{M_{W}^{6}} I
\end{aligned}
$$

where $I$ and $J$ are dimensionless integrals, with values $\approx 1$ in the limit $M_{\Delta^{ \pm \pm \pm}} \gg M_{W}$ and $M_{\Delta^{ \pm \pm \pm}} \gg \Gamma_{\Delta^{ \pm \pm \pm}}$. The decay phase diagram of $\Delta^{ \pm \pm \pm}$is shown in Fig. 16 for
$M_{\Delta^{ \pm \pm \pm}}=400 \mathrm{GeV}$. We see from Fig. 16 that $l l W$ decays of $\Delta^{ \pm \pm \pm}$dominate for $v_{\Delta}<3.1 \times 10^{-5}\left(3.6 \times 10^{-5}\right) \mathrm{GeV}$ and the $W W W$ decay dominates otherwise for $\mathrm{NH}(\mathrm{IH})$. Similar to $\Delta^{ \pm \pm}$decay, neglecting the dimension-5 contribution in the couplings of Eq. (5.9) will move the crossover point by $17 \%$ in $v_{\Delta}$ to the higher side. The mass splitting has a minimal impact on the decay phase diagrams.

Since $\Delta^{ \pm \pm \pm}$decays to three-body final states for $\Delta M \geq 0$, its proper decay length is expected to be very large as confirmed by Fig. 17. For the range of $\Delta^{ \pm \pm \pm}$mass that is not excluded by EWPT, $c \tau$ can be as large as a few millimeters. However, for heavier masses, it falls sharply, as expected. Similar to $\Delta^{ \pm \pm}, c \tau$ is maximum for a value of $v_{\Delta}$ where the transition happens from $l l W$ dominated decay to $W W W$ dominated decay of $\Delta^{ \pm \pm \pm}$. In general, the effect of mass splitting is marginal since in the $\Delta M \geq 0$ case
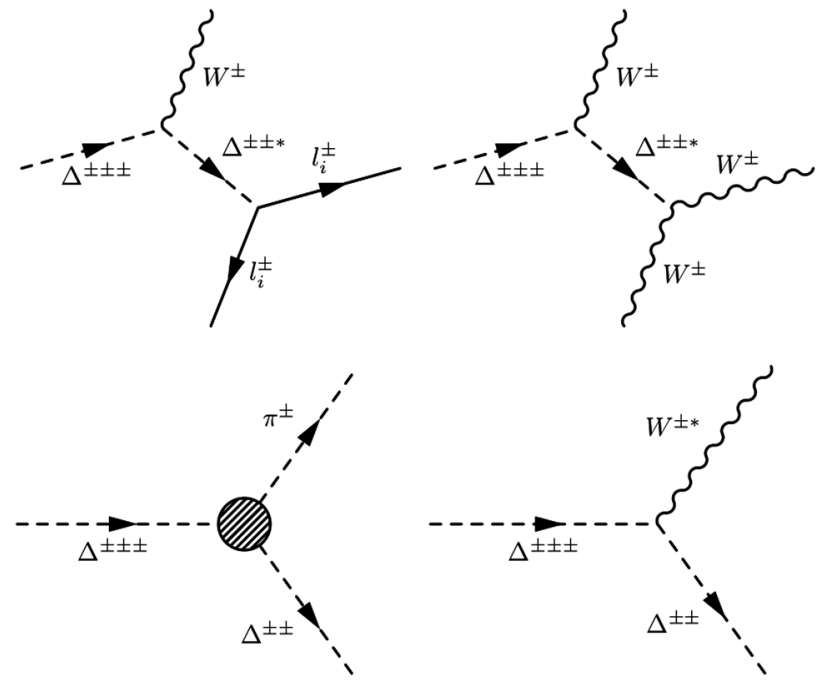

FIG. 15. Feynman diagrams for decay of $\Delta^{ \pm \pm \pm}$. The top two diagrams are for $\Delta M>0$, and the bottom two diagrams are for $\Delta M<0$. 

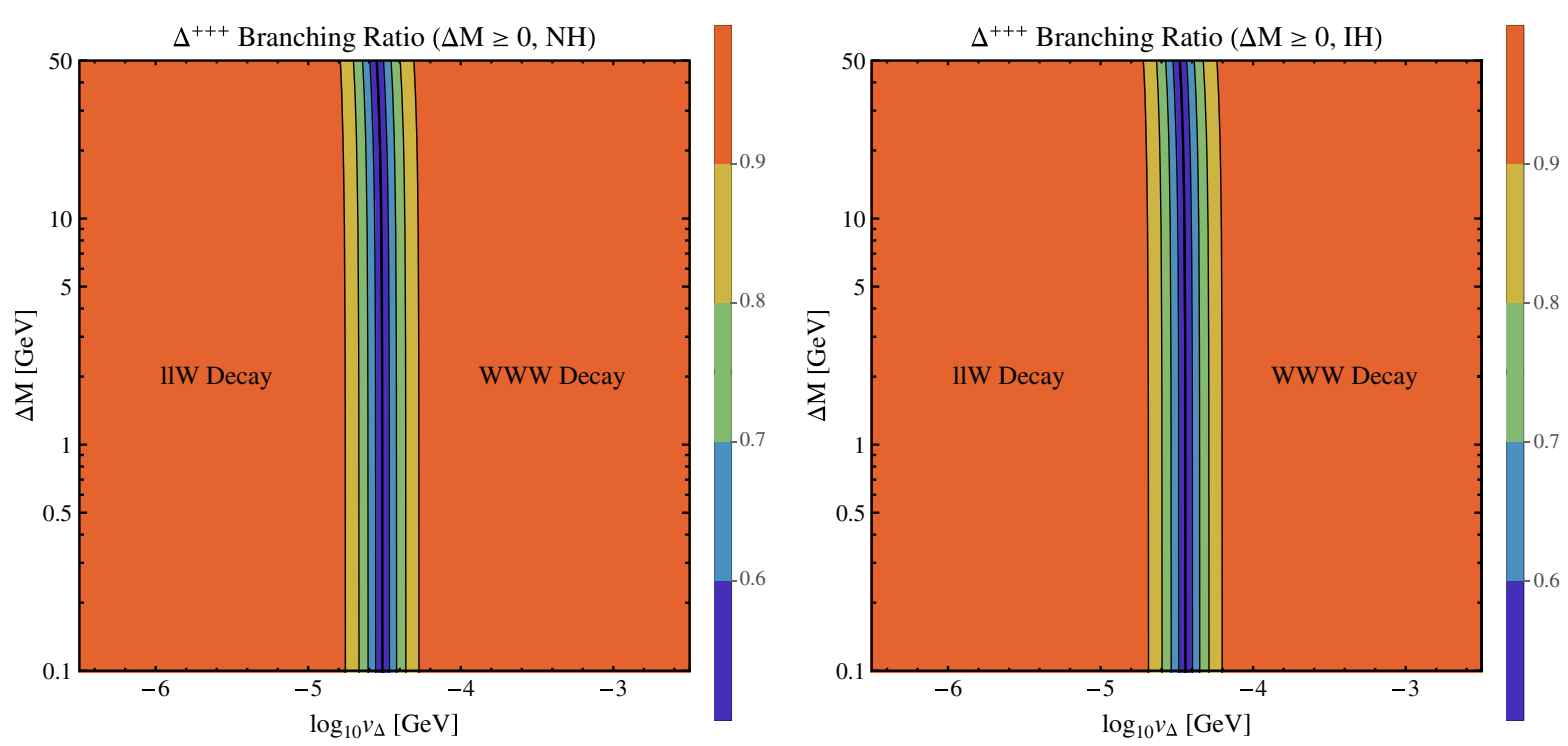

FIG. 16. Generic decay phase diagram for $\Delta^{ \pm \pm \pm}$decays in the BNT model, with $M_{\Delta^{ \pm \pm \pm}}=400 \mathrm{GeV}$ and $\Delta M \geq 0$, for both NH (left) and IH (right) of neutrino masses.

$\mathrm{NH}$

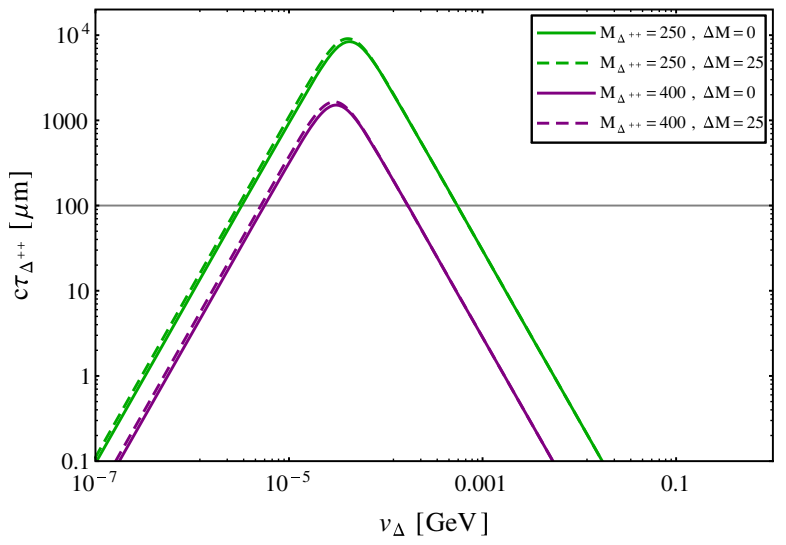

$\mathrm{IH}$

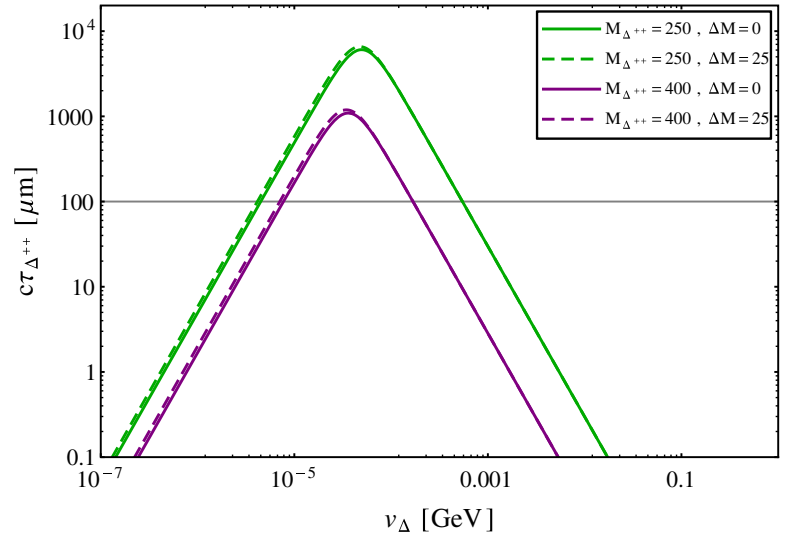

FIG. 17. Proper decay length of $\Delta^{ \pm \pm \pm}$for different values of $M_{\Delta^{ \pm \pm \pm}}$and $\Delta M$ for both NH (left) and IH (right) of neutrino masses. The gray horizontal lines in both panels refer to the limiting value of $c \tau$, up to which prompt-lepton searches at the LHC remain sensitive.

$\Delta^{ \pm \pm \pm}$is the lightest member of the quadruplet and no cascade channel is available. Nonetheless, it can change the decay length marginally in the $l l W$ dominated region due to the mass splitting entering in dimension-5 contribution to Yukawa couplings via $\Delta^{0}$ and $\Delta^{ \pm}$mass. Thus, we can infer beyond any reasonable doubt that for a large range of parameter space where $l l W$ and $W W W$ decay widths are commensurable $\Delta^{ \pm \pm \pm}$will elude any prompt lepton search at the LHC. In contrast, for the $\Delta M<0$ scenario, $\Delta^{ \pm \pm \pm}$ always decay via cascade, and such channels have large decay width, which makes them less interesting.

\section{Searches for $\Delta^{ \pm \pm}$at the LHC}

The LHC experiments have been searching for the doubly charged Higgs boson for some time. The CMS
Collaboration has made public their latest Run-II analysis with $12.9 \mathrm{fb}^{-1}$ [47] of data. With $36.1 \mathrm{fb}^{-1}$ [49] of data, ATLAS offers similar exclusion limits. Two crucial aspects of the CMS analysis are that it only considers scenarios where $\Delta M=0$ and also assumes that $\Delta^{ \pm \pm}$decays $100 \%$ to a particular flavor combination of $l^{ \pm} l^{ \pm}$. Reference [12] also uses LHC Run-I data to impose bounds on $\Delta^{ \pm \pm}$in the context of the BNT model. However, in a realistic scenario, consistent with available neutrino mass and mixing data, no leptonic channel will have a $100 \%$ BR. Hence, the novelty of our analysis is to take into account a benchmark for both $\mathrm{NH}$ and IH, as outlined in Sec. II D, and investigate how the limits relax in each case.

CMS conducted its search for doubly charged Higgs in exactly the $3 l$ final state for its associated production with a singly charged Higgs. In the BNT model, $\Delta^{ \pm \pm}$can also be 
produced in association with $\Delta^{ \pm \pm \pm}$, which can potentially double the production cross section. However, for this channel, $\Delta^{ \pm \pm \pm} \rightarrow l^{ \pm} l^{ \pm} W^{ \pm}\left(\Delta^{ \pm \pm} W^{* \pm}\right)$ decay for the $\Delta M>$ $0(\Delta M<0)$ case will give rise to extra leptons in the final state, and they will not pass the additional lepton-veto criteria of the CMS analysis. In contrast, pair production of $\Delta^{ \pm \pm}$for the $\Delta M>0$ case mentioned above will be sensitive to this study if one lepton is lost or mistagged, but given the range of $M_{\Delta^{ \pm \pm}}$we are interested in, the occurrence of such events is very unlikely. This is because the decay of $\Delta^{ \pm \pm}$leads to appreciably energetic leptons [50], which has high tagging efficiency.

On the other hand, for the $4 l$ study, CMS does not require any veto on additional leptons. So, for this final state, not only will the pair production of $\Delta^{ \pm \pm}$contribute, but also in the $\Delta M<0$ case, the pair production of $\Delta^{ \pm \pm \pm}$ will assist. Therefore, the limits drawn from this study will have some asymmetry between $\Delta M<0$ and $\Delta M>0$ cases. Another important point we need to address for pair productions of the doubly and triply charged Higgs bosons is whether to include PF in deriving the limits or not. As mentioned previously, we choose to adopt a conservative approach in this paper and use DY only for our calculation due to large uncertainties associated with the photon PDF.

Also, when $\Delta^{ \pm \pm}$dominantly decays in cascade, it can easily give rise to three or four leptons in the final state. However, such leptons will come from off-shell $W$ bosons, and the momentum they carry will have an upper bound of $\Delta M$. We have seen from Fig. 4 that EWPT bound limits $\Delta M \lesssim 30 \mathrm{GeV}$ for the most part of the range of $M_{\Delta^{ \pm \pm}}$we are studying. We need to juxtapose this limitation with the requirement of the CMS analysis that at least one lepton should have $p_{T}>30 \mathrm{GeV}$ and others should satisfy $p_{T}>20 \mathrm{GeV}$. Therefore, a tiny amount of cascade events will pass these hard cuts on lepton $p_{T}$. Furthermore, these soft leptons will not be able to reconstruct the narrow $M_{\Delta^{ \pm \pm}}$ mass peak, which is a criterion in the CMS analysis, due to significant momenta that will be carried away by missing neutrinos. Hence, we do not consider cascade decay products of $\Delta^{ \pm \pm}$in the subsequent computations. Interestingly, the compressed spectra are very similar to certain supersymmetric scenarios, well studied in the literature [51].

In Fig. 18, we plot the bounds derived from the CMS search of Ref. [47], on top of EWPT excluded regions in the $\Delta M-M_{\Delta^{ \pm \pm \pm}}$plane for $v_{\Delta}=10^{-6} \mathrm{GeV}$. This choice of $v_{\Delta}$ ensures that $\Delta^{ \pm \pm}$decays leptonically when $\Delta M=0$. The exclusion contours from the $3 l$ (left) and $4 l$ (right) final states are shown in the top panel for $\mathrm{NH}$ by cyan shaded regions. The bottom panel contains the same for IH. Additionally, we require $c \tau_{\Delta^{ \pm \pm}}<100 \mu \mathrm{m}$ so that the leptonic decay products are prompt. As mentioned earlier in the section, we consider DY production of $\Delta^{ \pm \pm}$only in the above figure. We should mention here that in Fig. 18 we only show the limits from the flavor combination decay channel that offers the strongest bound. So, for $\mathrm{NH}$ and $\mathrm{IH}$, we only show bounds derived from $\mu \mu$ and $e e$ channels, respectively. Although $\Delta^{ \pm \pm}$has a large BR to $\tau \tau$ decay for $\mathrm{NH}$, this channel does not impose strong bounds due to poor $\tau$ identification efficiency at the LHC. One may try to combine different channels, which will lead to an even stronger bound. However, we do not attempt to do that in this paper.

In general, the CMS search for $\Delta^{ \pm \pm}$using $12.9 \mathrm{fb}^{-1}$ integrated luminosity at $\sqrt{s}=13 \mathrm{TeV}$ bounds $M_{\Delta^{ \pm \pm}} \gtrsim$ $600 \mathrm{GeV}$ (at 95\% C.L.) for $\Delta M \lesssim 5 \mathrm{GeV}$ in the $3 l$ final state for both $\mathrm{NH}$ and IH. For the $4 l$ analysis, the bounds derived, using DY only, are $M_{\Delta^{ \pm \pm}} \gtrsim 600(400) \mathrm{GeV}$ for $\Delta M<0(\Delta M>0)$ for $\mathrm{IH}$. The bounds for $\mathrm{NH}$ are weaker by $\sim 50 \mathrm{GeV}$ compared to IH.

Figure 19 is the same as Fig. 18 but for $v_{\Delta}=5 \times 10^{-5} \mathrm{GeV}$. For such a value of $v_{\Delta}$, we have $\operatorname{BR}\left(\Delta^{ \pm \pm} \rightarrow l^{ \pm} l^{ \pm}\right) \approx \operatorname{BR}\left(\Delta^{ \pm \pm} \rightarrow W^{ \pm} W^{ \pm}\right)$, when cascade decay channels are not open. As expected, the bounds are relatively weak compared to the previous case. Interestingly, the bounds for $\mathrm{NH}$ and $\mathrm{IH}$ differ appreciably. From the $3 l$ analysis, we obtain a bound of $M_{\Delta^{ \pm \pm}} \gtrsim$ $400(500) \mathrm{GeV}$ for $\mathrm{NH}(\mathrm{IH})$, with $\Delta M \sim 0$. Similarly, from the $4 l$ final state, we get $M_{\Delta^{ \pm \pm}} \gtrsim 350(500) \mathrm{GeV}$ for $\mathrm{NH}$ (IH), again with $\Delta M \sim 0$. The difference between the $\mathrm{NH}$ and IH bounds is due to the fact that the crossover between dominantly $l l$ decay to dominantly $W W$ decay does not happen for the same $v_{\Delta}$ for them. So, for a choice of $v_{\Delta}$ for which $\operatorname{BR}\left(\Delta^{ \pm \pm} \rightarrow l^{ \pm} l^{ \pm}\right) \approx \mathrm{BR}\left(\Delta^{ \pm \pm} \rightarrow W^{ \pm} W^{ \pm}\right)$for $\mathrm{IH}$, the NH BP will be relatively in the $W W$ decay dominated region.

For a larger value of $v_{\Delta}$, the $W W$ BR will rapidly increase at the expense of $l l \mathrm{BR}$. Hence, the bounds derived from the CMS analysis of Ref. [47] for $v_{\Delta} \gtrsim 10^{-4} \mathrm{GeV}$ will be very weak and will be discussed elsewhere. No dedicated study by CMS or ATLAS exists for $\Delta^{ \pm \pm} \rightarrow W^{ \pm} W^{ \pm}$. However, Ref. [52] estimated a bound of $M_{\Delta^{ \pm \pm}}>84 \mathrm{GeV}$ for such decays using ATLAS Run-I results [53].

\section{E. Signal of $\Delta^{ \pm \pm \pm}$at the $\mathrm{LHC}$}

In the previous section, we discussed LHC studies that are searching for $\Delta^{ \pm \pm}$. However, $\Delta^{ \pm \pm}$is not exclusive to this model, and it may also arise in other models, such as the Georgi-Machacek model [54], littlest Higgs model [55], 3-3-1 models [56,57], Type-II seesaw models [6], left-right symmetric models [58,59], and radiative neutrino mass models [8]. Discovering/excluding $\Delta^{ \pm \pm}$alone will not identify/falsify the BNT model. In addition, from Figs. 18 and 19, we have noticed that the LHC can constrain $M_{\Delta^{ \pm \pm}}$for $\Delta M<5 \mathrm{GeV}$ only. Hence, to search for $\Delta^{ \pm \pm \pm}$directly at the LHC is imperative for the validation of the BNT model. 

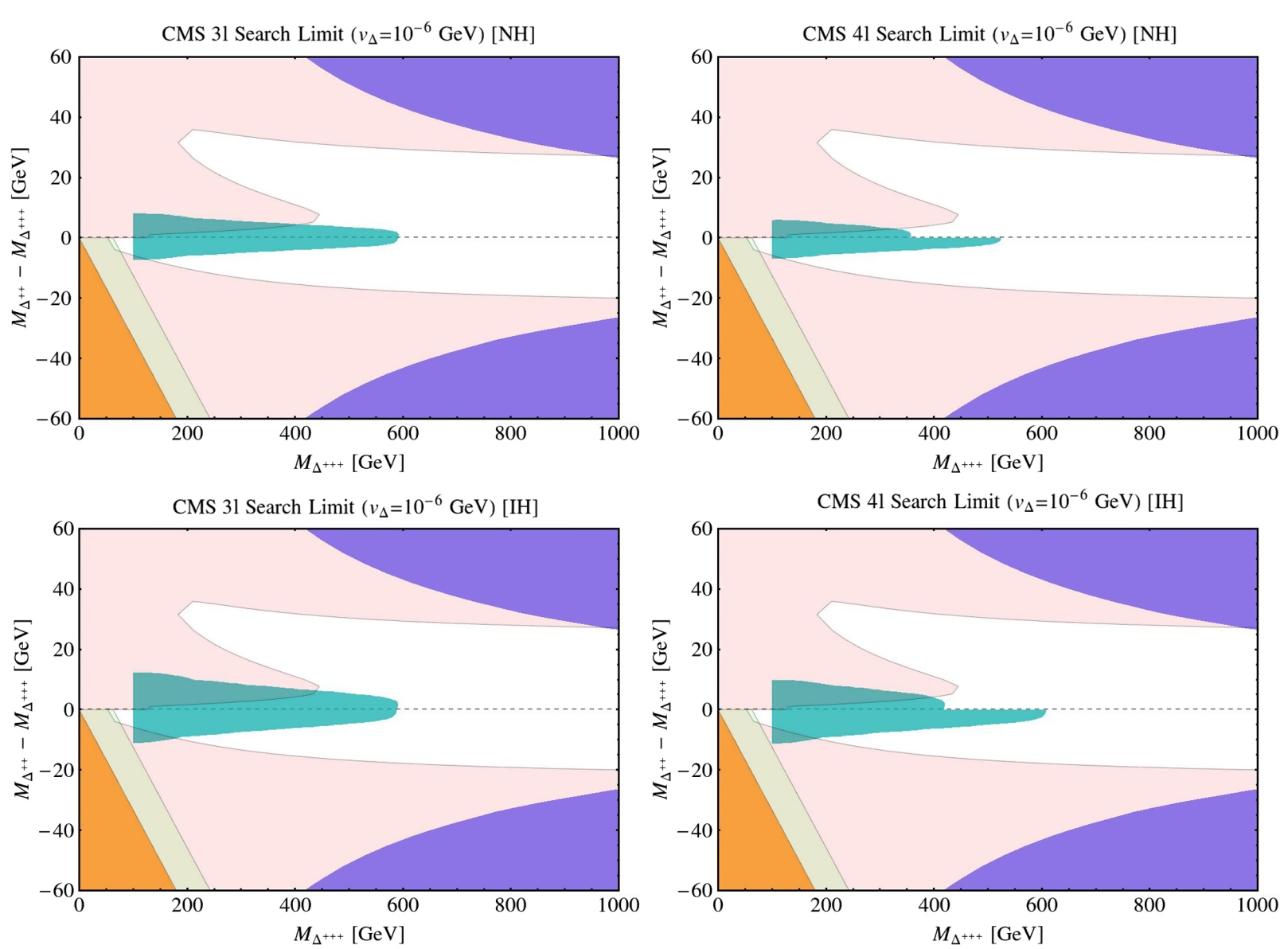

FIG. 18. Constraints form CMS searches for $\Delta^{ \pm \pm}$using $12.9 \mathrm{fb}^{-1}$ integrated luminosity at $\sqrt{s}=13 \mathrm{TeV}$. $v_{\Delta}$ is fixed at $10^{-6} \mathrm{GeV}$ so that $\Delta^{ \pm \pm}$decays leptonically when $\Delta M=0$. We show the limits derived from $3 l$ search (top left) and $4 l$ search (top right) for NH by cyan shaded regions. The two figures in the bottom panel are the same for IH. We also impose $c \tau_{\Delta^{ \pm \pm}}<100 \mu \mathrm{m}$. The bounds derived for $\mathrm{NH}(\mathrm{IH})$ are from the $\mu \mu(e e)$ decay channel. Only DY production is considered in the figure. The other colored regions have the same meaning as Fig. 4.

In this section, we present a feasibility study of the potential reach of the LHC in the search for $\Delta^{ \pm \pm \pm}$. We search for $\Delta^{ \pm \pm \pm}$in the same-sign (SS) $3 l(l=e, \mu)$ final state. We have already mentioned that the BNT model is implemented with the FEYNRULES_V2.0 [44] package. The signal and background events are generated using the MADGRAPH5_AMC@NLO_V2.5.4 code [43] followed by showering and hadronization by PYTHIA_V8.2 [60] and the detector simulation by DELPHES_V3.3 [61]. We produce $\Delta^{ \pm \pm \pm}$by a combination of $p p \rightarrow \Delta^{ \pm \pm \pm} \Delta^{\mp \mp \mp}+$ $\Delta^{ \pm \pm} \Delta^{\mp \mp}+\Delta^{ \pm \pm \pm} \Delta^{\mp \mp}$ processes.

The major SM backgrounds for our signal are $\bar{t} \bar{t} W^{ \pm}+$jets. However, $W^{ \pm} Z+$ jets and $Z / \gamma^{*}\left(\rightarrow l^{+} l^{-}\right) Z+$ jets may also contribute in the case of mismeasurement of the charge of a lepton. The latter backgrounds, in fact, dominate over the former since their production cross sections are significantly higher. $t \bar{t} Z\left(\gamma^{*}\right)+$ jets, $t \bar{t} b \bar{b}$, and $t \bar{t} t \bar{t}$ will also contribute, but they are much smaller compared to $t \bar{t} W^{ \pm}$[11], and we neglect them in our analysis. All the backgrounds are generated including up to one parton. The MLM scheme [62] for jet-parton matching has been employed to avoid double counting. For the backgrounds, $W$ and $Z$ bosons and top quarks are decayed in their respective leptonic decay channels with the MADSPIN [63] module of MADGRAPH5. In contrast, for the signal samples, the multicharged Higgs bosons has been decayed within PYTHIA. We perform all cross section calculations at tree level and do not include any $K$-factor. Therefore, our estimates for signal significance will likely be conservative. We use the default DELPHES3.3 detector card for various object reconstructions, with jet clustering performed using the anti-kt algorithm. The above detector card employs the following lepton and $b$-quark reconstruction criteria:

(i) Lepton identification and efficiency.-Electrons and muons are identified for $p_{T}>10 \mathrm{GeV}$ with $|\eta|<2.4$. While the electron efficiency is $85 \%$ and $95 \%$ for $|\eta|<1.5$ and $1.5<|\eta|<2.4$, respectively, the muon efficiency is kept constant at $95 \%$ over the whole pseudorapidity range. 

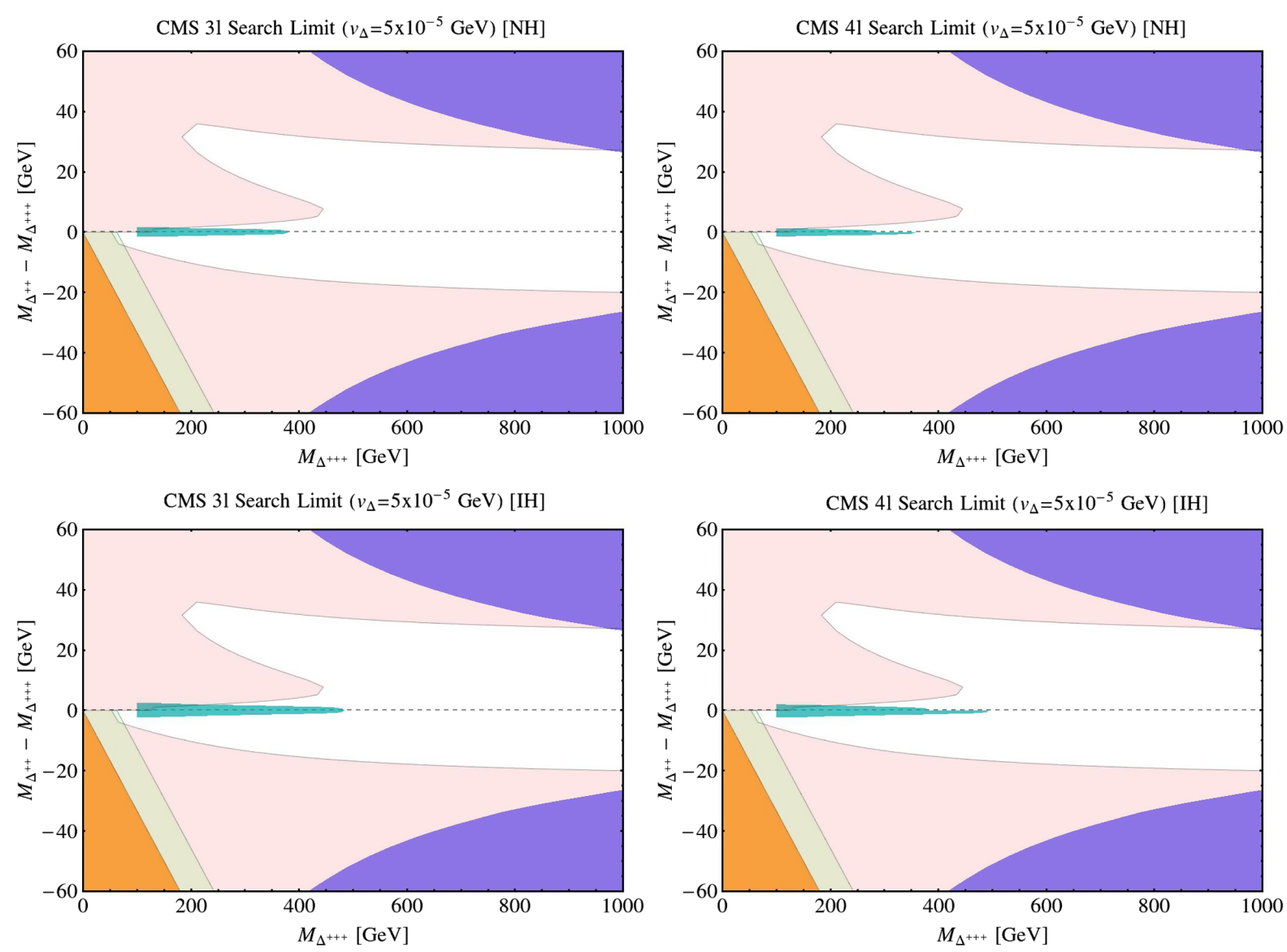

FIG. 19. Constraints form CMS searches for $\Delta^{ \pm \pm}$using $12.9 \mathrm{fb}^{-1}$ integrated luminosity at $\sqrt{s}=13 \mathrm{TeV}$. $v_{\Delta}$ is fixed at $5 \times 10^{-5} \mathrm{GeV}$ so that $\Delta^{ \pm \pm}$decays to a pair of leptons or gauge bosons with equal BR when $\Delta M=0$. We show the limits derived from the $3 l$ search (top left) and $4 l$ search (top right) for $\mathrm{NH}$ by the cyan shaded region. The two figures in the bottom panel are the same for IH. We also impose $c \tau_{\Delta^{ \pm \pm}}<100 \mu \mathrm{m}$. The bounds derived for $\mathrm{NH}(\mathrm{IH})$ are from the $\mu \mu(e e)$ decay channel. Only DY production is considered in the figure. The other colored regions have the same meaning as Fig. 4.

(ii) Lepton isolation.- - Lepton isolation is parametrized by $I_{\text {rel }}<0.25(0.12)$ for $\mu(e)$, where $I_{\text {rel }}$ is the ratio of the sum of transverse momenta of isolation objects (tracks, calorimeter towers, etc.) within a $\Delta R=\sqrt{(\Delta \eta)^{2}+(\Delta \phi)^{2}}=0.5$ cone around a candidate and the candidate's transverse momentum.

(iii) $b$-tagging efficiency.-The $b$-tagging efficiency is just above $70 \%$ for transverse momenta between 85 and $250 \mathrm{GeV}$, with a mistag rate $\lesssim 2 \%$, coming from $u, d, c, s, g$ jets, over the same energy range.

Next, using the above reconstructed objects, we list the selection cuts used in our SS $3 l$ study. They are:

(1) Basic cuts.-The signal and background events are preselected with the requirement of $p_{T_{l}(j)}>$ $10(20) \mathrm{GeV}$ and $\left|\eta_{l(j)}\right|<2.4(5)$. The subsequent cuts applied on the preselected events are optimized to maximize the signal significance, $S / \sqrt{S+B}$, where $S$ and $B$ denote signal and background rates.

(2) $\geq 3$ SS leptons. - We select events with at least three isolated SS light leptons $(e, \mu)$.
(3) Lepton $p_{T}$ cuts.-We impose the following stringent $p_{T}$ cuts on the selected SS leptons: $p_{T_{l_{1}}}>30 \mathrm{GeV}$, $p_{T_{l_{2}}}>30 \mathrm{GeV}$ and $p_{T_{l_{3}}}>20 \mathrm{GeV}$.

(4) Missing energy cut.-The missing energy cut is not very effective for the signal process after applying the hard lepton $p_{T}$ cuts. The $p_{T}$ cuts force the QCD radiation into a regime where jets produce a fair amount of missing energy as well. Hence, we enforce a nominal $E_{\mathrm{T}}^{\prime}>30 \mathrm{GeV}$.

(5) Z-veto.- If leptons having a charge opposite that of the three tagged leptons are present in an event, we veto such an event if any opposite-sign same flavor lepton pair combination satisfies $80 \mathrm{GeV}<M_{l^{ \pm} l^{\mp}}<100 \mathrm{GeV}$.

(6) b-veto.-We veto any events with one or more identified $b$-tagged jets, with $p_{T}>20 \mathrm{GeV}$ and $|\eta|<2.5$.

Table III gives the signal and background cross sections at $\sqrt{s}=14 \mathrm{TeV}$ after applying each cut listed above, accompanied by corresponding statistical errors. For the 
TABLE III. Summary of the signal and the background cross sections and corresponding statistical errors at our chosen benchmark point, after each kinematical cut, for NH of neutrino masses. The LHC center-of-mass energy is 14 TeV. In the first row, all background cross sections are presented after decaying top quarks and $W$ and $Z$ bosons in their respective leptonic channels within MADSPIN.

\begin{tabular}{|c|c|c|c|c|c|}
\hline$\left(M_{\Delta^{ \pm \pm \pm}}, \Delta M, v_{\Delta}\right) \mathrm{GeV}$ & Selection cuts & Signal (fb) & $W Z+$ jets $(\mathrm{fb})$ & $Z l^{+} l^{-}+$jets $(\mathrm{fb})$ & $t \bar{t} W+$ jets $(\mathrm{fb})$ \\
\hline \multirow{5}{*}{$\left(400,0,10^{-6}\right)$} & Basic cuts & $23.35 \pm 0.1044$ & $1167 \pm 1.948$ & $155.5 \pm 0.2596$ & $24.41 \pm 0.0446$ \\
\hline & $\geq 3$ SS leptons & $1.670 \pm 0.0279$ & $0.0975 \pm 0.0178$ & $0.0347 \pm 0.0039$ & $0.0044 \pm 0.0006$ \\
\hline & Lepton $p_{T}$ cuts & $1.443 \pm 0.0260$ & $0.0227 \pm 0.0086$ & $0.0087 \pm 0.0019$ & $0.0017 \pm 0.0004$ \\
\hline & $Z$-veto & $1.2847 \pm 0.0245$ & $0.0130 \pm 0.0065$ & $0.0039 \pm 0.0013$ & $0.0015 \pm 0.0003$ \\
\hline & $b$-veto & $1.1946 \pm 0.0236$ & $0.0130 \pm 0.0065$ & $0.0039 \pm 0.0013$ & $0.0003 \pm 0.0002$ \\
\hline
\end{tabular}

signal, we choose a BP with $\left(M_{\Delta^{ \pm \pm \pm}}, \Delta M, v_{\Delta}\right)=$ $\left(400,0,10^{-6}\right) \mathrm{GeV}$ for $\mathrm{NH}$ of neutrino masses. $v_{\Delta}$ is chosen to be $10^{-6} \mathrm{GeV}$ to ensure $\operatorname{BR}\left(\Delta^{ \pm \pm \pm} \rightarrow\right.$ $\left.l^{ \pm} l^{ \pm} W^{ \pm}\right)=1$, when $\Delta^{ \pm \pm \pm}$is the lightest member of the quadruplet. Here, we use $14 \mathrm{TeV}$ of center-of-mass energy as opposed to $13 \mathrm{TeV}$ used in previous subsections. This is due to the fact that we intend to estimate the discovery potential of $\Delta^{ \pm \pm \pm}$not only at an immediately achievable integrated luminosity of $100 \mathrm{fb}^{-1}$ but also at a high luminosity of $3000 \mathrm{fb}^{-1}$. The LHC is expected to run at $14 \mathrm{TeV}$ for that high luminosity benchmark. Elevating the center-of-mass energy to $14 \mathrm{TeV}$ for our simulation leads to an increase in the overall cross section of $p p \rightarrow$ $\Delta^{ \pm \pm \pm} \Delta^{\mp \mp \mp}+\Delta^{ \pm \pm} \Delta^{\mp \mp}+\Delta^{ \pm \pm} \Delta^{\mp \mp}$ processes by $\sim 20 \%$. Clearly, Table III indicates that the final state we are studying is almost devoid of SM background for our chosen BP. We do not show the effect of the $E_{\mathrm{T}}$ cut in the above cut-flow table since both signal and background have $\sim 100 \%$ efficiency for that cut.

Figure 20 shows the invariant mass of the three leading SS leptons and $E_{\mathrm{T}}$ for the signal with $\left(M_{\Delta^{ \pm \pm \pm}, \Delta M}\right)=$ $(400,0) \mathrm{GeV}$ for $\mathrm{NH}$. We set $v_{\Delta}=10^{-6} \mathrm{GeV}, 6 \times 10^{-5} \mathrm{GeV}$, and $5 \times 10^{-3} \mathrm{GeV}$ to achieve $\operatorname{BR}\left(\Delta^{ \pm \pm \pm} \rightarrow l^{ \pm} l^{ \pm} W^{ \pm}\right)=1$,

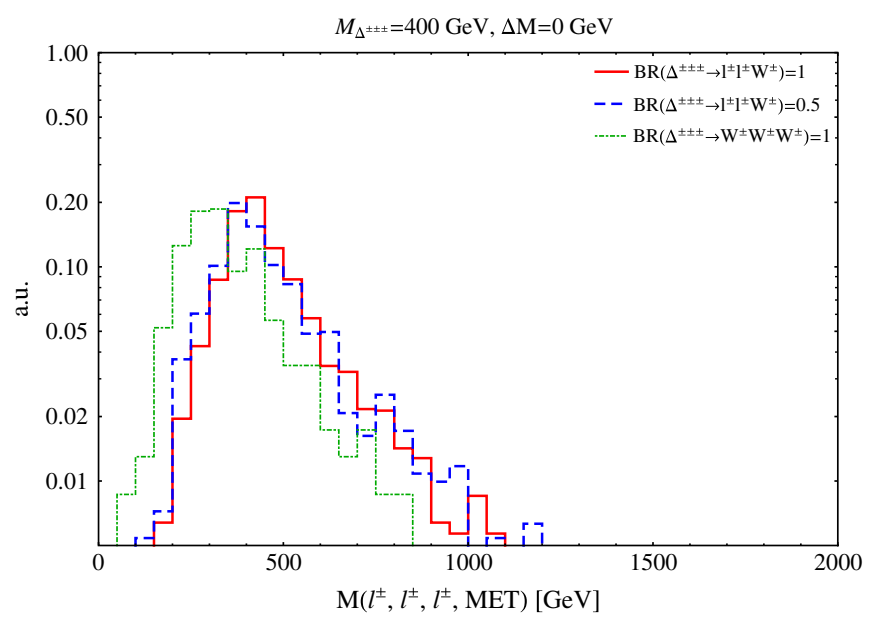

FIG. 20. The invariant mass of the three leading SS light leptons and $E_{\mathrm{T}}$ for the signal, after all the kinematic cuts. We keep $\left(M_{\Delta^{ \pm \pm \pm}}, \Delta M\right)=(400,0) \mathrm{GeV}$ fixed for three distinct BR scenarios. The $\mathrm{BP}$ is chosen for $\mathrm{NH}$ of neutrino masses.
$\operatorname{BR}\left(\Delta^{ \pm \pm \pm} \rightarrow l^{ \pm} l^{ \pm} W^{ \pm}\right)=0.5, \quad$ and $\quad \operatorname{BR}\left(\Delta^{ \pm \pm \pm} \rightarrow\right.$ $\left.W^{ \pm} W^{ \pm} W^{ \pm}\right)=1$, respectively. While we see a peak close to but not exactly at $M_{\Delta^{ \pm \pm \pm}}$for the first two cases, the peak is shifted significantly to a lower mass for the third case due to a large fraction of momentum carried by neutrinos coming from three $W$ decays.

In Fig. 21, we present $5 \sigma$ discovery reaches of $\Delta^{ \pm \pm \pm}$ at $\sqrt{s}=14 \mathrm{TeV}$ for integrated luminosities 100 and $3000 \mathrm{fb}^{-1}$. We show the mass reach for both $\mathrm{NH}$ (left panel) and $\mathrm{IH}$ (right panel) of neutrino masses for $v_{\Delta}=10^{-6} \mathrm{GeV}$. Also, for this value of $v_{\Delta}, c \tau_{\Delta^{ \pm \pm \pm}} \lesssim$ $100 \mu \mathrm{m}$ is definitely satisfied (cf. Fig. 17). The difference in mass reaches for $\mathrm{NH}$ and $\mathrm{IH}$ is minimal. We find that at $5 \sigma$ level $M_{\Delta^{ \pm \pm \pm}}$can be probed up to $\sim 600 \mathrm{GeV}$ for $100 \mathrm{fb}^{-1}$ and $\sim 950 \mathrm{GeV}$ with $3000 \mathrm{fb}^{-1}$.

Figure 22 is the same as Fig. 21 but for $v_{\Delta}=5 \times$ $10^{-3} \mathrm{GeV}$ that simultaneously ensures $\operatorname{BR}\left(\Delta^{ \pm \pm \pm} \rightarrow\right.$ $\left.W^{ \pm} W^{ \pm} W^{ \pm}\right)=1$ for $\Delta M>0$, and $c \tau_{\Delta^{ \pm \pm \pm}} \lesssim 100 \mu \mathrm{m}$ (cf. Fig. 17). The discovery potentials of $M_{\Delta^{ \pm \pm \pm}}$at the LHC are $\sim 325 \mathrm{GeV}$ and $\sim 600 \mathrm{GeV}$ with 100 and $3000 \mathrm{fb}^{-1}$ of integrated luminosities, respectively. We do not show a separate plot for $\operatorname{BR}\left(\Delta^{ \pm \pm \pm} \rightarrow l^{ \pm} l^{ \pm} W^{ \pm}\right)=$ $\operatorname{BR}\left(\Delta^{ \pm \pm \pm} \rightarrow W^{ \pm} W^{ \pm} W^{ \pm}\right)=0.5$ cases as most of the parameter space that can be probed at $100 \mathrm{fb}^{-1}$ will possess $c \tau_{\Delta^{ \pm \pm \pm}} \gtrsim 100 \mu \mathrm{m}$ and will not respond to our prompt lepton search strategy. Nonetheless, $3000 \mathrm{fb}^{-1}$ of integrated luminosity will offer a discovery reach of $M_{\Delta^{ \pm \pm \pm}} \sim$ 500-900 GeV for $\Delta M \geq 0$. One important point to notice is that we cover the entire $\Delta M \geq 0$ range allowed by EWPT in all cases.

One common feature of both Figs. 21 and 22 is that our SS $3 l$ search strategy is sensitive to a mass splitting of $\lesssim 10 \mathrm{GeV}$ when $\Delta^{ \pm \pm \pm}$is the heaviest member of the quadruplet. In those scenarios, cascade decay of $\Delta^{ \pm \pm \pm}$ will give rise to soft leptons that will not pass through our strong lepton $p_{T}$ cuts. A dedicated study with boosted topologies is needed for this kind of mass spectra, similar in flavor to compressed supersymmetric spectra studies [51]. One might use the Bayesian optimization techniques, as recently outlined in Ref. [64], for a systematic study of compressed spectra.

Finally, a comment is in order to distinguish $\mathrm{NH}$ and $\mathrm{IH}$ scenarios. The best way to distinguish them is to probe 

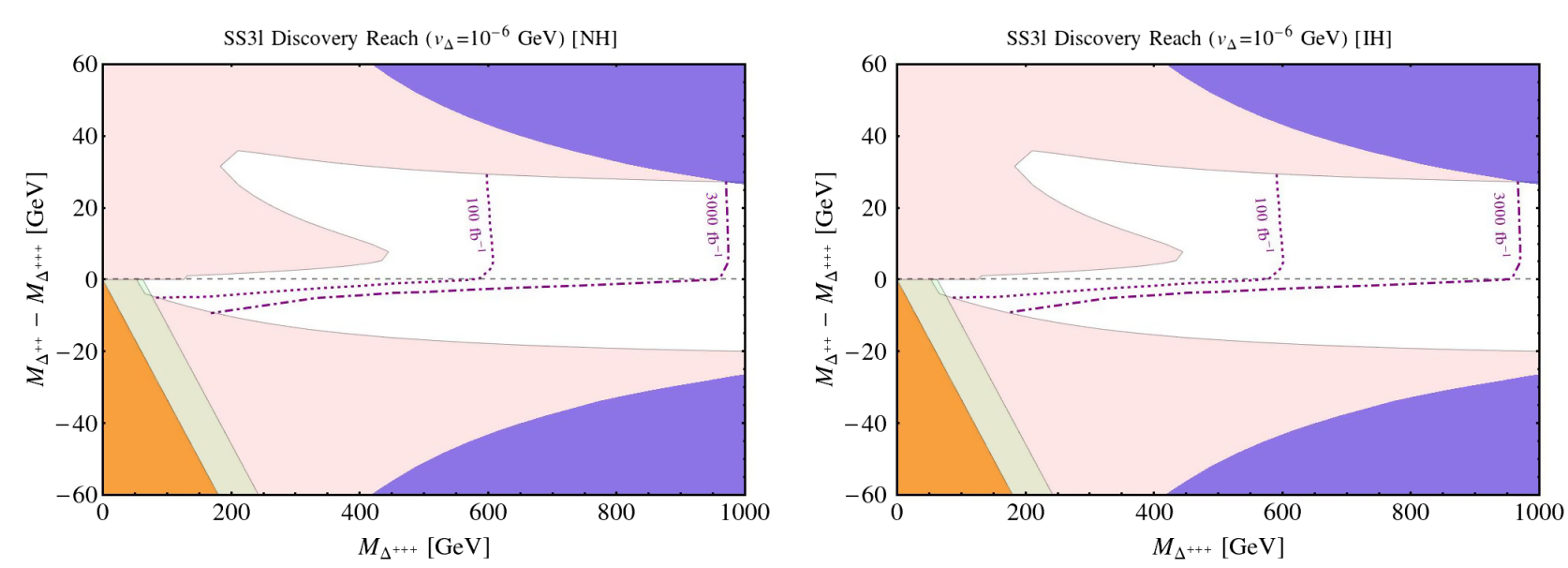

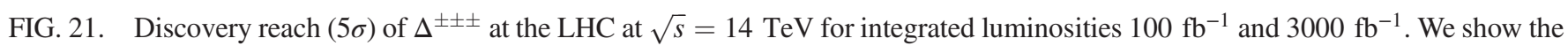
mass reach for both $\mathrm{NH}$ (left) and $\mathrm{IH}$ (right) of neutrino masses. $v_{\Delta}$ is set at $10^{-6} \mathrm{GeV}$ to ensure $\mathrm{BR}\left(\Delta^{ \pm \pm \pm} \rightarrow l^{ \pm} l^{ \pm} W^{ \pm}\right)=1$ for $\Delta M>0$. The other colored regions have the same meaning as Fig. 4.
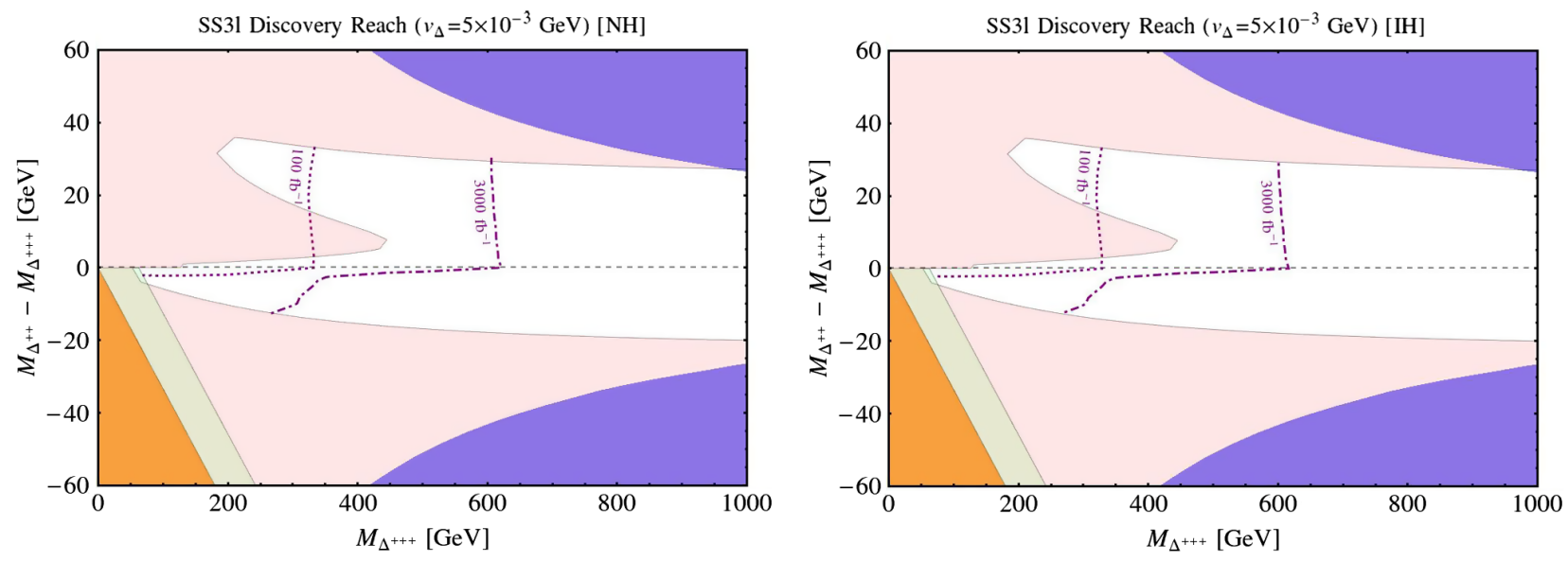

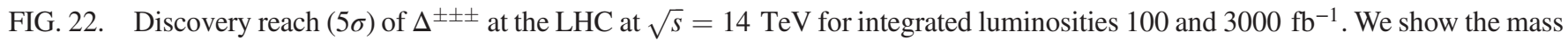
reach for both $\mathrm{NH}$ (left) and $\mathrm{IH}$ (right) of neutrino masses. $v_{\Delta}$ is set at $5 \times 10^{-3} \mathrm{GeV}$ to ensure $\mathrm{BR}\left(\Delta^{ \pm \pm \pm} \rightarrow W^{ \pm} W^{ \pm} W^{ \pm}\right)=1$ for $\Delta M>0$. The other colored regions have the same meaning as Fig. 4.

different flavor combinational leptonic decay channels of $\Delta^{ \pm \pm}$. We refer the reader to Ref. [46] for a detailed study on this, also including the impact of Dirac and Majorana phases. However, as it is made clear earlier in our analysis of Sec. V D, any search of $\Delta^{ \pm \pm}$is futile for $\Delta M \gtrsim 5 \mathrm{GeV}$ in the context of this model. Our SS $3 l$ search of this section can, on the other hand, probe all $\Delta M \geq 0$ mass spectra, but the total signal yield for both $\mathrm{NH}$ and $\mathrm{IH}$ is very similar. For example, for our chosen BP of $\left(M_{\Delta^{ \pm \pm \pm}}, \Delta M, v_{\Delta}\right)=$ $\left(400,0,10^{-6}\right) \mathrm{GeV}$, we expect to produce 119 and 111 events at $100 \mathrm{fb}^{-1}$ for $\mathrm{NH}$ and $\mathrm{IH}$. However, one needs to classify these SS $3 l$ events in different lepton flavor combinations to compare $\mathrm{NH}$ and $\mathrm{IH}$ more meticulously. A comparison, in that spirit, is presented in Table IV for the above $\mathrm{BP}^{3}$ The experimentally measured neutrino mixing angles imply that the heaviest neutrino mass state contains a tiny fraction of $\nu_{e}$ for $\mathrm{NH}$. Thus, one would expect very few events involving $e$ compared to $\mu$, as reflected in Table IV. In contrast, for $\mathrm{IH}$, the more massive neutrino mass states have large $\nu_{e}$ and $\nu_{\mu}$ components. Therefore, a

\footnotetext{
${ }^{3}$ We should point out here that the flavor combinations of leptons in SS $3 l$ signal shown in Table IV are calculated assuming all $C P$ phases to be 0 . However, nonzero $C P$-violating phases in the Pontecorvo-Maki-Nakagawa-Sakata (PMNS) matrix can alter the above flavor combination breakdown. Nonetheless, the most important distinguishing factor in that table, namely, the ratio of $\mu \mu \mu /$ eee events, will remain similar regardless of $C P$ violating phase choices.
} 
TABLE IV. Neutrino mass hierarchy dependency in the SS $3 l$ signal in the llW dominant region for the neutrino mass and mixing parameters chosen in Table II including vanishing $C P$ phases.

\begin{tabular}{lrrrcc}
\hline \hline SS $3 l$ & $e e e$ & $e e \mu$ & $e \mu \mu$ & $\mu \mu \mu$ & Total events \\
\hline NH & 1 & 9 & 62 & 47 & 119 \\
IH & 31 & 54 & 14 & 12 & 111 \\
\hline \hline
\end{tabular}

comparable number of $e$ and $\mu$ events is expected in this case, which can again be noticed from Table IV. Although the lepton flavor combinations of SS $3 l$ final state events are more or less reflective of neutrino mixing hierarchies, one should also keep in mind that $e, \mu$ identification efficiencies and energy resolutions differ, but they are expected to have a minimal impact on our analysis due to strong $p_{T}$ cuts used.

\section{CONCLUSIONS}

We study various phenomenological implications of a dimension-7 neutrino mass generation mechanism, as proposed in the BNT model [9], in this paper. The model contains an isospin $3 / 2$ scalar quadruplet $(\Delta)$ and two vectorlike isotriplet leptons $\left(\Sigma_{1,2}\right)$, in addition to the $\mathrm{SM}$ field content. We reiterate the claim of Ref. [9] that one can get light neutrino masses, consistent with observed oscillation parameters, with $\mathcal{O}(\mathrm{TeV})$-scale new physics. Although the dimension-7 operator develops neutrino masses at tree level, the model cannot prevent dimension-5 operator contributions to the same at loop level. In fact, one needs to set $M_{\Sigma} \lesssim 1 \mathrm{TeV}$ to probe dimension-7 operator contribution explicitly, but a such choice of parameters leads us to a very computationally expensive regime without any new insight into the Higgs sector of the model. Hence, we integrate out $\Sigma_{1,2}$ by setting $M_{\Sigma}=5 \mathrm{TeV}$ and work with the resulting effective Lagrangian. For this choice of $M_{\Sigma},\left(m_{\nu}\right)_{i j}^{\text {loop }}$ and $\left(m_{\nu}\right)_{i j}^{\text {tree }}$ are comparable for the range of $M_{\Delta}$ accessible to the ongoing run of the LHC. Loop contributions will dominate for higher values of $M_{\Sigma}$.

One novel feature of our paper is a high precision electroweak study of the model. It is well known that the EW $\rho$ parameter constrains the induced VEV obtained by the quadruplet, $v_{\Delta} \lesssim 1 \mathrm{GeV}$. However, we probe the model more closely for its contribution to the oblique parameters and estimate the impact of them on the quadruplet mass spectrum. Over the range of $M_{\Delta}$ that is accessible to the LHC, the most robust constraint comes from the $T$ parameter, which is controlled by the mass splitting, $\Delta M$, between the quadruplet members. We find that EWPT limits $\Delta M \lesssim 30 \mathrm{GeV}$, which in turn give rise to compressed spectra over a vast area of the parameter space. Due to the softness of decay products in a compressed scenario, a significant part of the parameter space will remain beyond the reach of the LHC, when $\Delta M<0$.

Next, we investigate the unique signatures of the model at the LHC. The presence of multicharged scalars of the model can potentially enhance or suppress the $h \rightarrow \gamma \gamma$ decay rate depending on the sign of the coupling. Using $36 \mathrm{fb}^{-1}$ data from both CMS and ATLAS, we deduce that $h \rightarrow \gamma \gamma$ can exclude regions of parameter space not ruled out by EWPT, albeit for $\mathcal{O}(1)$ values of $\lambda_{3}$. For smaller $\lambda_{3}$, it does not add anything to EWPT. We also consider the bounds from the $\mu \rightarrow e \gamma$ LFV process on our parameter space and derive a lower bound on $v_{\Delta} \sim \mathcal{O}(1 \mathrm{eV})$ from $M_{\Delta^{ \pm \pm}} \lesssim 1 \mathrm{TeV}$. Mass splitting between $\Delta$ components, or ordering of neutrino masses, has a negligible impact on the above limit.

We also examine the BRs and proper decay lengths of $\Delta^{ \pm \pm}$ and $\Delta^{ \pm \pm}$in detail, along with their consequences at the LHC, for the whole range of $\Delta M$ allowed by EWPT. We find that for $\Delta^{ \pm \pm}$cascade decays start to dominate for $\Delta M \sim$ 2-20 GeV for both signs of $\Delta M$. In contrast, for $\Delta^{ \pm \pm \pm}$, no cascade decay is available when $\Delta M \geq 0$, but it always decays in cascade for $\Delta M<0$. A large $c \tau$ is achievable for both $\Delta^{ \pm \pm}$and $\Delta^{ \pm \pm \pm}$when $v_{\Delta} \sim 10^{-5}-10^{-4} \mathrm{GeV}$ and $\Delta M \sim 0$. In this region, the leptonic and gauge bosonic decay rates of $\Delta^{ \pm \pm}$are comparable, and $c \tau$ can be as large as $10 \mu \mathrm{m}$, which is still within the realm of prompt lepton searches at the LHC. Similarly, for $\Delta^{ \pm \pm \pm}$, a transition from $l l W$ dominated decay to $W W W$ dominated decay happens around that region, and $c \tau \gtrsim 100 \mu \mathrm{m}$ is feasible for $M_{\Delta^{ \pm \pm \pm}} \lesssim$ $500 \mathrm{GeV}$ and as a result forces this region to be insensitive to prompt lepton searches at the LHC. However, when cascade decay opens up, the proper decay length increases rapidly and brings $\Delta^{ \pm \pm \pm}$within the reach of the LHC.

A strong bound on $M_{\Delta^{ \pm \pm}}$can be derived from $3 l$ and $4 l$ searches performed by the CMS Collaboration with $12.9 \mathrm{fb}^{-1}$ data. The strongest bounds are obtained when $\operatorname{BR}\left(\Delta^{ \pm \pm} \rightarrow l^{ \pm} l^{ \pm}\right)=1$, which we ensure by setting $v_{\Delta}=10^{-6} \mathrm{GeV}$. We extract the limits from the leptonic decay channel that provides the best sensitivity for a particular ordering of neutrino masses, which is $\mu \mu$ for $\mathrm{NH}$ and $e e$ for $\mathrm{IH}$ for our chosen neutrino mass and mixing benchmark values. Using the CMS $3 l$ analysis, we constrain $M_{\Delta^{ \pm \pm}} \gtrsim 600 \mathrm{GeV}$. The limits on $\Delta^{ \pm \pm}$mass fall sharply as $\operatorname{BR}\left(\Delta^{ \pm \pm} \rightarrow l^{ \pm} l^{ \pm}\right)$deviates from 1 . Moreover, the above bounds are sensitive for $|\Delta M|<5 \mathrm{GeV}$ only.

Finally, we perform a feasibility study to examine the discovery reach of $\Delta^{ \pm \pm \pm}$at the LHC. A search for $\Delta^{ \pm \pm \pm}$is necessary, independent of $\Delta^{ \pm \pm}$searches conducted by the LHC experiments, to validate the BNT model, as $\Delta^{ \pm \pm}$is not unique to this model. Furthermore, the LHC multilepton searches for $\Delta^{ \pm \pm}$is not sensitive for a large mass gap. In contrast, a direct search for $\Delta^{ \pm \pm \pm}$can cover the whole range of $\Delta M$, allowed by EWPT for $\Delta M>0$. A simple set of cuts, led by hard cuts on $p_{T}$ of leptons, is sufficient to isolate the $\mathrm{SS} 3 l$ signature that can arise from $\Delta^{ \pm \pm \pm}$decay. With $3 \mathrm{ab}^{-1}$ of integrated luminosity, the LHC can discover $\Delta^{ \pm \pm \pm}$for a mass up to 950 (600) GeV in the $l l W(W W W)$ decay dominant regions for both $\mathrm{NH}$ and IH of neutrino masses. 
Nevertheless, the search strategy used in our analysis will not be effective for $\Delta M<0$ scenarios. In these cases, $\Delta^{ \pm \pm \pm}$will predominantly decay via cascade, and the decay products will not pass the hard lepton $p_{T}$ cuts we used here. A dedicated analysis is needed to probe such mass spectra in the flavor of compressed SUSY spectra studies.

\section{ACKNOWLEDGMENTS}

We thank Kaladi Babu, Teruki Kamon, Luca Pernie, Bhupal Dev, and Xerxes Tata for useful comments. T. G. has been supported in part by the US Department of Energy Grant No. de-sc 0016013 and is now supported by NSF CAREER Grant No. PHY-1250573. S. N. and S. J. have been supported in part by the US Department of Energy Grant No. de-sc 0016013. The work of S. J. is also supported in part by the Fermilab Distinguished Scholars Program. S. J. thanks the Fermilab Theoretical Physics Department for warm hospitality during the completion of this work.

\section{APPENDIX A: EXPANSION OF LAGRANGIANS IN TENSOR NOTATION}

The field $\Delta$ has component fields $\Delta=\left(\Delta^{+++}, \Delta^{++}, \Delta^{+}\right.$, $\left.\Delta^{0}\right)^{T}$. In tensor notation, $\Delta$ is a total symmetric tensor $\Delta_{i j k}$, with three indices $i, j, k$ taking values 1 and 2 . Therefore, we can write various components of $\Delta$ as

$\Delta_{111}=\Delta^{+++}, \quad \Delta_{112}=\frac{\Delta^{++}}{\sqrt{3}}, \quad \Delta_{122}=\frac{\Delta^{+}}{\sqrt{3}}, \quad \Delta_{222}=\Delta^{0}$.

$\Sigma_{1,2}$ are symmetric tensors alike, with two indices, and they can be written in tensor notation as

$\Sigma_{i_{11}}=\Sigma_{i}^{++}, \quad \Sigma_{i_{12}}=\frac{\Sigma_{i}^{+}}{\sqrt{2}}, \quad \Sigma_{i_{22}}=\Sigma_{i}^{0} \quad(i=1,2)$.

Hence, in terms of component fields, the last term of the scalar potential of Eq. (2.3) is given by

$$
\begin{aligned}
H^{3} \Delta^{*} & =H_{a} H_{b} H_{c} \Delta^{*^{a b c}} \\
& =\phi^{+^{3} \Delta^{---}}+3 \phi^{+^{2}} \phi^{0} \frac{\Delta^{--}}{\sqrt{3}}+3 \phi^{+} \phi^{0^{2}} \frac{\Delta^{-}}{\sqrt{3}}+\phi^{0^{3}} \Delta^{0} .
\end{aligned}
$$

Similarly, the Yukawa terms in Eq. (2.10) can be expanded as

$$
\begin{aligned}
\overline{L_{i L}{ }^{c}} H^{*} \Sigma_{1}= & \left(\overline{L_{i L}{ }^{c}}\right)_{a} H^{*^{b}} \Sigma_{1_{b c}} \epsilon^{a c} \\
= & \overline{\nu_{i L}}\left(\phi^{-} \frac{\Sigma_{1}^{+}}{\sqrt{2}}+\phi^{0^{*}} \Sigma_{1}^{0}\right) \\
& -\overline{{l_{i L}}^{c}}\left(\phi^{-} \Sigma_{1}^{++}+\phi^{0^{*}} \frac{\Sigma_{1}^{+}}{\sqrt{2}}\right),
\end{aligned}
$$

$$
\begin{aligned}
\overline{\Sigma_{2}} \Delta L_{i L}= & {\overline{\Sigma_{2}}}^{a b} \Delta_{a b c}\left(L_{i L}\right)_{d} \epsilon^{c d} \\
= & \left(\Sigma_{2}^{--} \Delta^{+++}+2 \frac{\Sigma_{2}^{-}}{\sqrt{2}} \frac{\Delta^{++}}{\sqrt{3}}+\Sigma_{2}^{0^{*}} \frac{\Delta^{+}}{\sqrt{3}}\right) l_{i L}^{-} \\
& -\left(\Sigma_{2}^{--} \frac{\Delta^{++}}{\sqrt{3}}+2 \frac{\Sigma_{2}^{-}}{\sqrt{2}} \frac{\Delta^{+}}{\sqrt{3}}+\Sigma_{2}^{0^{*}} \Delta^{0}\right) \nu_{i L},
\end{aligned}
$$

where $\epsilon^{a b}=\left(\begin{array}{cc}0 & 1 \\ -1 & 0\end{array}\right)$ is a totally antisymmetric tensor. Finally, we present the effective Lagrangian of Eq. (2.11) in terms of component fields,

$$
\begin{aligned}
\overline{L_{i L}{ }^{c}} L_{j L} H^{*} \Delta= & \left(\overline{L_{i L}{ }^{c}}\right)_{a} L_{j L} a^{\prime} H^{* b} \Delta_{b c d} \epsilon^{a c} \epsilon^{a^{\prime} d} \\
= & \overline{\nu_{i L}{ }^{c}} \nu_{j L}\left(\phi^{-} \frac{\Delta^{+}}{\sqrt{3}}+\phi^{0 *} \Delta^{0}\right) \\
& -\overline{\bar{l}_{i L}{ }^{c}} \nu_{j L}\left(\phi^{-} \frac{\Delta^{++}}{\sqrt{3}}+\phi^{0 *} \frac{\Delta^{+}}{\sqrt{3}}\right) \\
& -\overline{\nu_{i L}} l_{j L}^{-}\left(\phi^{-} \frac{\Delta^{++}}{\sqrt{3}}+\phi^{0 *} \frac{\Delta^{+}}{\sqrt{3}}\right) \\
& +\overline{\overline{l i L}^{-}} l_{j L}^{-}\left(\phi^{-} \Delta^{+++}+\phi^{0 *} \frac{\Delta^{++}}{\sqrt{3}}\right) .
\end{aligned}
$$

\section{APPENDIX B: FEYNMAN RULES RELEVANT FOR $\boldsymbol{\Delta}^{ \pm \pm}$AND $\boldsymbol{\Delta}^{ \pm \pm \pm}$INTERACTIONS}

The couplings relevant for the production and decay of doubly and triply charged scalars are shown in Table V. A factor 2 is included whenever two identical particles are in the vertex. Two such examples are $\Delta^{ \pm \pm} W^{\mp} W^{\mp}$ and $\Delta^{ \pm \pm} l_{i}^{\mp} l_{j}^{\mp}$ (for $i=j$ ). Also, we consider the $C P$-violating phases of the PMNS matrix to be 0 for our BPs. Hence, for our study, $\left(m_{\nu}\right)_{i j}^{\text {tot }}=\left(m_{\nu}\right)_{j i}^{\text {tot }}$, and a factor of 2 is included for $\Delta^{ \pm \pm} l_{i}^{\mp} l_{j}^{\mp}($ for $i \neq j)$ as well.

TABLE V. Feynman rules relevant for the production and decay of doubly and triply charged scalars of the BNT Model. Here, $p_{i}$ stands for the 4-momentum of the $i$ th particle at the vertex, with the convention that all the particle momenta are coming into the vertex. For brevity, $\cos 2 \theta_{W}\left(\sin 2 \theta_{W}\right)$ has been abbreviated as $c_{2 W}\left(s_{2 W}\right)$. In the last interaction, $D$ is given in Eq. (5.10).

\begin{tabular}{lc}
\hline \hline Vertex & Couplings \\
\hline$A^{\mu} \Delta^{ \pm \pm \pm} \Delta^{\mp \mp \mp}$ & $-3 i e\left(p_{2}-p_{3}\right)_{\mu}$ \\
$A^{\mu} \Delta^{ \pm \pm} \Delta^{\mp \mp}$ & $-2 i e\left(p_{2}-p_{3}\right)_{\mu}$ \\
$Z^{\mu} \Delta^{ \pm \pm \pm} \Delta^{\mp \mp \mp}$ & $-\frac{3 i \cos 2 \theta_{w}}{\sin 2 \theta_{w}}\left(p_{2}-p_{3}\right)_{\mu}$ \\
$Z^{\mu} \Delta^{ \pm \pm} \Delta^{\mp \mp}$ & $-\frac{2 i e\left(\cos 2 \theta_{w}-1 / 2\right)}{\sin 2 \theta_{w}}\left(p_{2}-p_{3}\right)_{\mu}$ \\
$W^{\mu \mp} \Delta^{\mp \mp} \Delta^{ \pm \pm \pm}$ & $-i \sqrt{\frac{3}{2}} g\left(p_{2}-p_{3}\right)_{\mu}$ \\
$W^{\mu \mp} \Delta^{\mp} \Delta^{ \pm \pm}$ & $-i \sqrt{2} g\left(p_{2}-p_{3}\right)_{\mu}$ \\
$\Delta^{ \pm \pm} W^{\mp} W^{\mp}$ & $\sqrt{6} g^{2} v_{\Delta} g_{\mu \nu}$ \\
$\Delta^{ \pm \pm} l_{i}^{\mp} l_{j}^{\mp}$ & $\frac{2}{\sqrt{6}} \frac{\left(m_{\nu} \operatorname{lot}_{i j}\right.}{D}$ \\
\hline \hline
\end{tabular}


[1] G. Aad et al. (ATLAS Collaboration), Phys. Lett. B 716, 1 (2012).

[2] S. Chatrchyan et al. (CMS Collaboration), Phys. Lett. B 716, 30 (2012).

[3] P. A. R. Ade et al. (Planck Collaboration), Astron. Astrophys. 571, A16 (2014).

[4] S. Weinberg, Phys. Rev. Lett. 43, 1566 (1979).

[5] P. Minkowski, Phys. Lett. 67B, 421 (1977); T. Yanagida, Conf. Proc. C 7902131, 95 (1979); M. Gell-Mann, P. Ramond, and R. Slansky, Conf. Proc. C 790927, 315 (1979); R. N. Mohapatra and G. Senjanovic, Phys. Rev. Lett. 44, 912 (1980).

[6] M. Magg and C. Wetterich, Phys. Lett. 94B, 61 (1980); J. Schechter and J. W. F. Valle, Phys. Rev. D 22, 2227 (1980); C. Wetterich, Nucl. Phys. B187, 343 (1981); G. Lazarides, Q. Shafi, and C. Wetterich, Nucl. Phys. B181, 287 (1981); R. N. Mohapatra and G. Senjanovic, Phys. Rev. D 23, 165 (1981).

[7] R. Foot, H. Lew, X. G. He, and G. C. Joshi, Z. Phys. C 44, 441 (1989); E. Ma, Phys. Rev. Lett. 81, 1171 (1998).

[8] A. Zee, Nucl. Phys. B264, 99 (1986); K. S. Babu, Phys. Lett. B 203, 132 (1988).

[9] K. S. Babu, S. Nandi, and Z. Tavartkiladze, Phys. Rev. D 80, 071702 (2009).

[10] M. Chala, C. Krause, and G. Nardini, arXiv:1802.02168.

[11] G. Bambhaniya, J. Chakrabortty, S. Goswami, and P. Konar, Phys. Rev. D 88, 075006 (2013).

[12] K. Ghosh, S. Jana, and S. Nandi, J. High Energy Phys. 03 (2018) 180.

[13] S. Bhattacharya, S. Jana, and S. Nandi, Phys. Rev. D 95, 055003 (2017).

[14] K. Ghosh, S. Jana, and S. Nandi, arXiv:1607.01910.

[15] Y. Liao, G. Z. Ning, and L. Ren, Phys. Rev. D 82, 113003 (2010).

[16] J. Adam et al. (MEG Collaboration), Phys. Rev. Lett. 110, 201801 (2013).

[17] P. S. B. Dev, C. M. Vila, and W. Rodejohann, Nucl. Phys. B921, 436 (2017).

[18] C. Patrignani et al. (Particle Data Group), Chin. Phys. C 40, 100001 (2016).

[19] K. Kannike, Eur. Phys. J. C 72, 2093 (2012).

[20] I. P. Ivanov, M. Kpke, and M. Mhlleitner, arXiv:1802 .07976 .

[21] K. Kannike, Eur. Phys. J. C 76, 324 (2016).

[22] G. F. Giudice and O. Lebedev, Phys. Lett. B 665, 79 (2008).

[23] M. Bauer, M. Carena, and K. Gemmler, J. High Energy Phys. 11 (2015) 016.

[24] F. Bonnet, D. Hernandez, T. Ota, and W. Winter, J. High Energy Phys. 10 (2009) 076.

[25] I. Gogoladze, N. Okada, and Q. Shafi, Phys. Lett. B 672, 235 (2009).

[26] R. Cepedello, M. Hirsch, and J. C. Helo, J. High Energy Phys. 07 (2017) 079; 01 (2018) 009.

[27] S. Davidson, G. Isidori, and A. Strumia, Phys. Lett. B 646, 100 (2007).

[28] M. C. Gonzalez-Garcia, M. Maltoni, and T. Schwetz, Nucl. Phys. B908, 199 (2016).

[29] M.E. Peskin and T. Takeuchi, Phys. Rev. D 46, 381 (1992).

[30] L. Lavoura and L. F. Li, Phys. Rev. D 49, 1409 (1994).
[31] M. Baak, M. Goebel, J. Haller, A. Hoecker, D. Kennedy, R. Kogler, K. Mönig, M. Schott, and J. Stelzer, Eur. Phys. J. C 72, 2205 (2012).

[32] G. Bambhaniya, P. S. B. Dev, S. Goswami, and M. Mitra, J. High Energy Phys. 04 (2016) 046.

[33] B. Ren, K. Tsumura, and X. G. He, Phys. Rev. D 84, 073004 (2011).

[34] A. Djouadi, Phys. Rep. 457, 1 (2008).

[35] S. Knapen, T. Melia, M. Papucci, and K. Zurek, Phys. Rev. D 93, 075020 (2016).

[36] S. Jana and S. Nandi, arXiv:1710.00619.

[37] A. Denner, S. Heinemeyer, I. Puljak, D. Rebuzzi, and M. Spira, Eur. Phys. J. C 71, 1753 (2011).

[38] S. Heinemeyer et al. (LHC Higgs Cross Section Working Group), arXiv:1307.1347.

[39] CMS Collaboration, CERN Report No. CMS-PAS-HIG-16040.

[40] ATLAS Collaboration, CERN Report No. ATLAS-CONF2017-045.

[41] K. S. Babu and S. Jana, Phys. Rev. D 95, 055020 (2017).

[42] R. D. Ball, V. Bertone, S. Carrazza, L. Del Debbio, S. Forte, A. Guffanti, N. P. Hartland, and J. Rojo (NNPDF Collaboration), Nucl. Phys. B877, 290 (2013).

[43] J. Alwall, R. Frederix, S. Frixione, V. Hirschi, F. Maltoni, O. Mattelaer, H.-S. Shao, T. Stelzer, P. Torrielli, and M. Zaro, J. High Energy Phys. 07 (2014) 079.

[44] A. Alloul, N. D. Christensen, C. Degrande, C. Duhr, and B. Fuks, Comput. Phys. Commun. 185, 2250 (2014).

[45] M. Aoki, S. Kanemura, and K. Yagyu, Phys. Rev. D 85, 055007 (2012).

[46] P. Fileviez Perez, T. Han, G. y. Huang, T. Li, and K. Wang, Phys. Rev. D 78, 015018 (2008).

[47] CMS Collaboration, Report No. CMS-PAS-HIG-16-036.

[48] https://indico.bnl.gov/getFile.py/access? contribId=15\& session $I d=5 \&$ resId $=0 \&$ materialId $=$ slides \& $\operatorname{confId}=1516$.

[49] M. Aaboud et al. (ATLAS Collaboration), Eur. Phys. J. C 78, 199 (2018).

[50] B. Dutta, R. Eusebi, Y. Gao, T. Ghosh, and T. Kamon, Phys. Rev. D 90, 055015 (2014).

[51] H. Baer, A. Mustafayev, and X. Tata, Phys. Rev. D 90, 115007 (2014); B. Dutta, A. Gurrola, W. Johns, T. Kamon, P. Sheldon, and K. Sinha, Phys. Rev. D 87, 035029 (2013); B. Dutta, T. Ghosh, A. Gurrola, W. Johns, T. Kamon, P. Sheldon, K. Sinha, K. Wang, and S. Wu, Phys. Rev. D 91, 055025 (2015); B. Dutta, K. Fantahun, A. Fernando, T. Ghosh, J. Kumar, P. Sandick, P. Stengel, and J. W. Walker, arXiv:1706.05339.

[52] S. Kanemura, M. Kikuchi, H. Yokoya, and K. Yagyu, Prog. Theor. Exp. Phys. 2015, 051B02 (2015).

[53] G. Aad et al. (ATLAS Collaboration), J. High Energy Phys. 03 (2015) 041.

[54] H. Georgi and M. Machacek, Nucl. Phys. B262, 463 (1985).

[55] N. Arkani-Hamed, A. G. Cohen, E. Katz, and A. E. Nelson, J. High Energy Phys. 07 (2002) 034.

[56] P. H. Frampton, Phys. Rev. Lett. 69, 2889 (1992).

[57] F. Pisano and V. Pleitez, Phys. Rev. D 46, 410 (1992).

[58] J. C. Pati and A. Salam, Phys. Rev. D 10, 275 (1974); R. N. Mohapatra and J. C. Pati, Phys. Rev. D 11, 566 (1975); 
G. Senjanovic and R. N. Mohapatra, Phys. Rev. D 12, 1502 (1975).

[59] R. Kuchimanchi and R. N. Mohapatra, Phys. Rev. D 48, 4352 (1993); K. S. Babu and R. N. Mohapatra, Phys. Lett. B 668, 404 (2008); L. Basso, B. Fuks, M. E. Krauss, and W. Porod, J. High Energy Phys. 07 (2015) 147.

[60] T. Sjstrand, S. Ask, J. R. Christiansen, R. Corke, N. Desai, P. Ilten, S. Mrenna, S. Prestel, C. O. Rasmussen, and P. Z. Skands, Comput. Phys. Commun. 191, 159 (2015).
[61] J. de Favereau, C. Delaere, P. Demin, A. Giammanco, V. Lemaître, A. Mertens, and M. Selvaggi (DELPHES 3 Collaboration), J. High Energy Phys. 02 (2014) 057.

[62] M. L. Mangano, M. Moretti, F. Piccinini, and M. Treccani, J. High Energy Phys. 01 (2007) 013.

[63] P. Artoisenet, R. Frederix, O. Mattelaer, and R. Rietkerk, J. High Energy Phys. 03 (2013) 015.

[64] A. Alves, T. Ghosh, and K. Sinha, Phys. Rev. D 96, 035022 (2017). 